\title{
MICROMAMÍFEROS, TAFONOMÍA Y PALEOAMBIENTES DEL CUATERNARIO TARDÍO EN TIERRA DEL FUEGO: LOS ROEDORES DE TRES ARROYOS 1
}

\author{
ULYSES F. J. PARDIÑAS ${ }^{a}$, FABIANA MARTIN ${ }^{b}$, LUIS A. BORRERO ${ }^{c}$, \\ MAURICIO MASSONE ${ }^{b} \&$ FERNANDO J. FERNÁNDEZ ${ }^{d}$
}

\section{RESUMEN}

Se estudiaron los restos de roedores recuperados en la secuencia estratigráfica del sitio arqueológico Tres Arroyos 1 (53 $53^{\circ} 23^{\prime} \mathrm{S}$, 68 $47^{\circ} \mathrm{O}$, Tierra del Fuego, Chile). Las muestras de niveles pleistocénicos (ca. 12,5-10 ka) están dominadas por Euneomys chinchilloides y Ctenomys magellanicus, con la única ocurrencia de Eligmodontia cf. E. morgani, un sigmodontino hoy día extinguido en la Isla Grande de Tierra del Fuego, pero con poblaciones en el extremo sur continental. Estos conjuntos sugieren paleoambientes hostiles, dominados por pedregales con escaso desarrollo de cobertura herbácea, bajo condiciones de frío intenso. En las muestras de los niveles del Holoceno tardío (ca. 1,3 ka-presente) son abundantes Reithrodon auritus y C. magellanicus y muestran una declinación de E. chinchilloides; se registran, además, especies que ocurren típicamente en la zona boscosa del sur de Tierra del Fuego (e.g. Abrothrix lanosa, Oligoryzomys longicaudatus). Estos conjuntos señalan un mejoramiento en las condiciones ambientales, acompañado de la implantación de estepas herbáceas y arbustivas y, quizás, expansión de la línea de bosque. Tafonómicamente, los agregados pleistocénicos revelan un origen mayoritario en acumulaciones generadas por mamíferos carniceros, mientras que aquellos holocénicos sugieren un origen múltiple en egagrópilas de aves rapaces nocturnas (particularmente para los restos de sigmodontinos) y consumo antrópico (para una parte de aquellos de Ctenomys). La integración de esta evidencia con aquella proveniente de otros archivos paleoclimáticos (e.g. polen) refleja una buena concordancia.

PALABRAS CLAVE: Ctenomys, Eligmodontia, Reithrodon, Euneomys, extinciones.

a Instituto de Diversidad y Evolución Austral (IDEAus - CONICET), Boulevard Brown 2915, 9120 Puerto Madryn, Chubut, Argentina e Investigador Asociado, Instituto Nacional de Biodiversidad (INABIO), Quito, Ecuador. ulyses@cenpat-conicet.gob. ar

b Centro de Estudios del Hombre Austral, Instituto de la Patagonia, Universidad de Magallanes, Av. Bulnes 01890, Punta Arenas, Chile. fabiana.martin@umag.cl; mauricio.massone@gmail.com

c Universidad de Buenos Aires e Instituto Multidisciplinario de Historia y Ciencias Humanas (IMHICIHU-CONICET), Saavedra 15 , piso $5^{\circ}$ (1083 ACA), Ciudad Autónoma de Buenos Aires, Argentina. laborrero2003@yahoo.com

d Grupo de Estudios en Arqueometría (GEA), Facultad de Ingeniería (UBA), Paseo Colón 1063, 850 Ciudad Autónoma de Buenos Aires, Argentina y Cátedra de Zooarqueología, Facultad de Ciencias Naturales y Museo, Universidad Nacional de La Plata, calle 64 s/n, 1900 La Plata, Buenos Aires. $\$ fernandezf77@yahoo.com.ar 


\title{
MICROMAMMALS, TAPHONOMY AND PALEOENVIRONMENTS OF THE LATE QUATERNARY AT TIERRA DEL FUEGO: THE RODENTS OF TRES ARROYOS 1
}

\begin{abstract}
We studied the micromammal remains recovered from the stratigraphic sequence of the

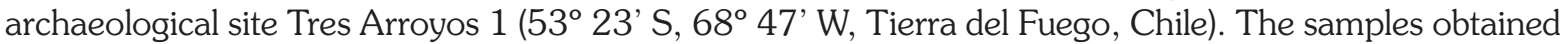
from Late Pleistocene levels (ca. 12.5-10 ky) are mainly composed by Euneomys chinchilloides and Ctenomys magellanicus, with the unique occurrence of Eligmodontia cf. E. morgani, a sigmodontine today extinct in the Isla Grande de Tierra del Fuego but with populations in the southernmost mainland. These assemblages suggest very hard paleoenvironments with a general rocky and bare landscape under deep cold and windy conditions. The Late Holocene samples (ca. 1.3 ky-present), by the contrary, are composed basically by Reithrodon auritus, C. magellanicus, some sigmodontine species typically from southern Tierra del Fuego forests (e.g. Oligoryzomys longicaudatus, Abrothrix lanosa), and show a declination of $E$. chinchilloides. These assemblages indicate amelioration in the environmental conditions surely coupled with the regional development of shrub and herbaceous steppes and perhaps a north displacement of the forest line. Taphonomically, pleistocenic assemblages seem to be mainly produced by the trophic activity of carnivore mammals, while those holocenic suggest a mixed origin with participation of owl pellets (especially for sigmodontine remains) and human consumption (responsible for a part of the Ctenomys remains). In overall, the micromammal evidence is in agreement with the results obtained from other paleoclimatic records (e.g. pollen).
\end{abstract}

KEY WORDS: Ctenomys, Eligmodontia, Reithrodon, Euneomys, extinctions.

\section{INTRODUCCIÓN}

El estudio de los micromamíferos en sitios arqueológicos puede brindar elementos de juicio relevantes para inferir los ambientes pretéritos. Sin embargo, para hacer de esto una realidad, es fundamental un adecuado control taxonómico, tafonómico y estratigráfico (e.g. Avery, 1982; Simonetti, 1989; Andrews, 1990, 1992; Hadly, 1996; Pardiñas, 1999a, 1999b).

Existen limitadas aproximaciones al estudio de los pequeños mamíferos que abarcan el intervalo entre el Pleistoceno tardío y la actualidad en Patagonia (e.g. Pearson, 1987; Pardiñas, 1999a; Pardiñas \& Teta, 2008, 2013; Fernández et al. 2012; Tammone et al. 2014; Andrade \& Fernández, 2017). En buena medida, esta escasez responde más a la dificultad del análisis, no así a la abundancia de este tipo de evidencias. De hecho, los restos de roedores y marsupiales son generalmente frecuentes en yacimientos bajo roca de la región patagónica (e.g. Simonetti \& Rau, 1989; Pearson \& Pearson, 1993; Pardiñas, 1999b; Hajduk et al. 2004; Reyes et al. 2007). Su valor como proxy data es de menor resolución si se lo compara con otros archivos paleoclimáticos usualmente estudiados (e.g. polen, restos macrobotánicos, moluscos, diatomeas, turberas). Sin embargo, al menos juzgando el volumen de la literatura de la última década, no parece manifestarse un interés creciente por los mismos.

La presencia de micromamíferos en yacimientos arqueológicos y paleontológicos de la Isla Grande de Tierra del Fuego (de aquí en más, Tierra del Fuego) ha sido destacada en algunas oportunidades (e.g. Latorre, 1998). También se han discutido sus potenciales procesos de incorporación a los contextos, especialmente atendiendo a la posibilidad de consumo antrópico (e.g. Borrero, 1979; Borrero \& Casiraghi, 1980; Pardiñas, 1999b; Pardiñas et al. 2011a; Santiago et al. 2016; Fernández et al. 2017a).

En este trabajo se estudian los conjuntos de micromamíferos recuperados durante las excavaciones del sitio Tres Arroyos 1 (de aquí en más, TA1). Se analizan los aspectos taxonómicos y tafonómicos (cualitativos y cuantitativos) de las muestras cronológicamente referidas al Pleistoceno más tardío y al Holoceno tardío. Con estas evidencias se genera una serie de hipótesis sobre 
los paleoambientes a nivel local y regional durante el depósito de los sedimentos portadores.

\section{MATERIALES Y MÉTODOS}

\section{Antecedentes generales del sitio}

TA1 (532 21' S, 68 48' O 92 msnm) se emplaza en la parte inferior de la ladera norte del cerro de Los Onas, un afloramiento rocoso formado por sedimentitas cenozoicas. Se trata de una cueva de $8,3 \mathrm{~m}$ de frente, $8,3 \mathrm{~m}$ de fondo $y$ 3,9 m de altura máxima. Su entrada está orientada en dirección noroeste y norte y domina el amplio valle, de origen glacial, que une bahía Inútil y bahía San Sebastián.

El sitio fue dado a conocer por Carlos Descourvieres, antiguo dueño de la Estancia Tres Arroyos. Tanto la Misión Francesa en la década de 1960, como Earl Saxon en 1975 practicaron sondeos limitados en su interior (LamingEmperaire, 1972; Saxon, 1979). Entre 1981 y 1999, con el apoyo del Instituto de la Patagonia $y$, posteriormente, a través de la UMAG y otras instituciones, uno de los autores y colaboradores llevaron a cabo excavaciones en TA1, que abarcaron una superficie total de $20 \mathrm{~m}^{2}$ (Massone, 1987, 2004; Massone et al. 1998).

Los depósitos más profundos del yacimiento, que corresponden a la capa VI, son portadores de fauna extinta; una datación sobre hueso de équido recuperado en este nivel indica $12.540 \pm 70$ años radiocarbónicos AP (Beta123152; 14.708 años calibrados AP, Mediana). En la capa Va se localizaron 5 fogones en cubeta, correspondientes a ocupaciones de cazadoresrecolectores del Pleistoceno final, con presencia de variados artefactos líticos, algunos artefactos óseos, restos de colorante y restos de fauna extinta y viviente. Las fechas más confiables obtenidas a partir de muestras de carbón de los fogones oscilan entre 10.130 y 10.855 años radiocarbónicos AP (rango comprendido entre 11.706 y 12.718 años calibrados AP, Medianas). Se trata de las ocupaciones humanas más antiguas detectadas en la cueva (Massone, 2004, 2010). Sucesivos estudios han mostrado que entre los vertebrados extinguidos presentes en los estratos inferiores de TA1 se registran caballo americano (Hippidion saldiasi), milodón (Mylodon darwini), diversos camélidos (Lama gracilis y un clado extinto de Lama guanicoe), félidos (Panthera onca mesembrina y Smilodon sp.), cánido (Dusicyon avus) y avestruces (Rheidae) (véase Caviglia, 1985-1986; Mengoni Goñalons, 1987; Prieto \& Canto, 1997; Latorre, 1998; Alberdi \& Prieto, 2000; Martin et al. 2009; Prevosti et al. 2013; Metcalf et al. 2016). También se ha discutido, de manera profusa, la génesis de estos depósitos y sus principales rasgos tafonómicos, donde se destaca la plausible acción de mamíferos carnívoros como formadores de parte del registro (e.g. Borrero, 2003; Massone, 2004; Martin, 2013; Prevosti et al. 2013).

En los niveles estratigráficos superiores de TA1, se encuentran a lo menos 4 eventos de ocupación humana, cuyos restos han quedado depositados en sedimentos arenosos de las capas IV, III, II y I. Corresponden a la presencia de grupos Selk'nam y sus antecesores, durante los últimos 1.300 años. En estos depósitos, donde se han preservado 9 fogones, predominan variados artefactos líticos, pequeños fragmentos de colorante rojo, algunos artefactos óseos y restos de distintas especies de fauna aún viviente, con predominio del guanaco (Lama guanicoe) y de roedores. Para el primero, numerosos restos muestran huellas que indican diversos estadios de procesamiento $y$ consumo final (Muñoz, 2000).

Los depósitos de TA1 presentan algunos sectores intactos o escasamente alterados, como es el caso de las estructuras de combustión referidas y algunas asociaciones contextuales. Sin embargo, diferentes sectores de la estratigrafía muestran importantes alteraciones por procesos postdepositacionales que han implicado migraciones verticales de algunos materiales óseos y, probablemente, líticos o la agregación de restos óseos por parte de carnívoros (Martin, 1997; Borrero, 2003).

\section{Contexto ambiental y \\ muestras de micromamiferos}

TA1 se emplaza en el paisaje de la sierra Carmen Sylva, penetración más nororiental de un pequeño sistema orográfico (con alturas máximas de ca. $300 \mathrm{msnm}$ ) limitado al norte por el sector 
deprimido situado entre bahía Inútil (Chile) y bahía San Sebastián (Argentina). Ambientalmente, corresponde a la porción esteparia o "patagónica" de Tierra del Fuego (Moore, 1983), que se extiende aproximadamente desde el curso del río Grande (ca. 53 54' S) hacia el estrecho de Magallanes (ca. $\left.52^{\circ} 40^{\prime} \mathrm{S}\right)$.

Los restos faunísticos del sitio, fundamentalmente aquellos de vertebrados medianos y grandes, han sido estudiados tanto en sus aspectos taxonómicos (e.g. Mengoni Goñalons, 1987; Prieto \& Canto, 1997; Latorre, 1998; Alberdi \& Prieto, 2000) como tafonómicos (Borrero, 2003; Martin, 2013). Para los pequeños mamíferos, Massone (1987) fue el primero en mencionar la presencia de Ctenomys en el yacimiento. Posteriormente, Latorre (1998) agregó la ocurrencia de los sigmodontinos Euneomys y Reithrodon.

Los restos de micromamíferos aquí estudiados corresponden a materiales extraídos durante las campañas de 1986 y 1996 de las cuadrículas A/B y D (véase una planta del sitio en Massone, 2004, p. 76 y Martin, 2013: Fig. 7.1). Fueron manualmente separados del conjunto de la arqueofauna que se conserva en las colecciones del Centro de Estudios del Hombre Austral, Instituto de la Patagonia, UMAG (Punta Arenas, Chile). Los datos estratigráficos son aquellos originalmente consignados por los excavadores en las etiquetas (véase Anexos 1 y 2). Se consideraron para el presente análisis las capas o niveles estratigráficos I, II, III, IV y Va de la cuadrícula A/B y II, III superior, IVa, IVb, Va, Vb y VI de la cuadrícula D. Aquellas divisiones estratigráficas de orden menor (e.g. capa Va base) fueron asimiladas en forma directa a la capa principal. Todos los materiales originalmente consignados como provenientes de áreas bioturbadas fueron determinados (Anexos 1 y 2), pero no contemplados en los cómputos finales o análisis de significación paleoambiental. Las determinaciones anatómicas y taxonómicas se efectuaron mediante comparación con colecciones de referencia del Centro Nacional Patagónico (Puerto Madryn, Chubut, Argentina). La asignación taxonómica -hasta el grado posible y siguiendo el criterio de Patton et al. (2015), excepto parcialmente para la taxonomía de Abrothrixfue efectuada solamente para elementos (sensu
Shotwell, 1955) craneanos, con un NISP total de 2.288 para la cuadrícula A/B (Anexo 1) y 2.377 para la D (Anexo 2). El postcráneo (NISP total $=5.018$ [A/B], 912 [D]) solo fue determinado anatómicamente y cuantificado a los fines de estimar abundancias absolutas y relativas; no se efectuaron correcciones mediante cálculo de MNE (sensu Lyman, 1994). Para cada muestra se calculó, además, la riqueza de especies (S).

Para cada conjunto se registraron variables tafonómicas básicas referidas al estado superficial del material y grado de completitud. Las variables de estado superficial fueron decisivas decisorias a la hora de establecer la autoctonía (i.e. el resto es coevo con el sedimento portador) o aloctonía (i.e. el resto es intrusivo respecto del sedimento portador) del material. Como detallara Massone (1987, 1988, véase también Borrero, 2003), la estratigrafía de TA1 presenta cierta complejidad, en particular por el grado de perturbación potencial atribuible a la acción de mamíferos excavadores en algunos sectores de los depósitos. La presencia de restos referibles a conejo europeo (Oryctolagus cuniculus) se verifica en casi todas las capas de TA1 (excepto capa VI, véanse Anexos 1 y 2), si bien con frecuencias minoritarias (Martin, 1997). Los restos de Oryctolagus presentan un estado superficial único entre toda la arqueofauna de TA1, caracterizado por un aspecto "fresco", coloración blanquecina, extrema fragilidad y porosidad. Seguramente, este mamífero fue el agente responsable de las madrigueras detectadas durante la excavación del yacimiento. Un rango estratigráfico que resultó particularmente vulnerado por la bioturbación fue la capa IV y el deslinde entre las capas IV y V (Massone, 1987, p. 51), sector estratigráfico clave por sus implicaciones cronológicas. En este contexto, diversos restos de la muestra aquí estudiada, se consideraron intrusivos; se trata mayormente de materiales que por sus características provienen de depósitos holocénicos (capas IV y suprayacentes), pero que fueron estratigráficamente recuperados en niveles pleistocénicos (capas V y VI; es decir, han descendido en la columna). Aun así cabe destacar que, especialmente para algunos restos de la capa V, la asociación estratigráfica no se puede considerar inequívoca, situación que solo parece soluble con fechados radiocarbónicos taxón. 
Sobre la base taxonómica cualitativa y cuantitativa se calcularon algunos de los índices tafonómicos propuestos por Pardiñas (1999a, 1999b). Las abundancias de elementos esqueletarios, transformadas a relativas (sensu Andrews, 1990: 45; $\mathrm{R}_{\mathrm{i}}=\mathrm{N}_{\mathrm{i}} / \mathrm{NISP}$, donde $\mathrm{N}_{\mathrm{i}}=$ frecuencia absoluta del elemento i) fueron evaluadas y comparadas con promedios de muestras actuales de aves rapaces nocturnas (Strigiformes), de rapaces diurnas (Accipitriformes y Falconiformes) y de mamíferos carnívoros (Felidae, Canidae, Mephitidae y Mustelidae) de América del Sur (Montalvo \& Fernández, 2019). Adicionalmente, se inspeccionaron los restos en busca de potenciales marcas de corrosión por acción de ácidos digestivos de depredadores, siguiendo los conceptos de Andrews (1990) y Fernández et al. (2017b). Se consideraron como atributos indicadores de explotación antrópica tanto el comportamiento y tamaño de las especies implicadas, los patrones de termoalteración y las marcas de corte (e.g. Pardiñas, 1999b; Fernández et al. 2017a y literatura alli citada).

Para evaluar la significación paleoambiental de los conjuntos, se analizaron los indicadores de presencia/ausencia y variaciones en las frecuencias (medidas según NISP por taxón y capa) en el contexto de las preferencias ambientales de los micromamíferos registrados (básicamente siguiendo a Osgood, 1943). La carencia de muestras comparables actuales (i.e. conjuntos actuales generados por la acción depredadora de búhos y lechuzas) a escala local o regional limitó la aplicación de análisis multivariados. Como indicadores de la presencia y, parcialmente, abundancia de los micromamíferos actuales en Tierra del Fuego, se tomaron datos generados por trampeos (e.g. Osgood, 1943; Patterson et al. 1984; Reise \& Venegas, 1987; Marconi, 1988; Massoia \& Chebez, 1993 y las referencias alli citadas).

Excepto indicación en contrario, todas las cronologías se brindan en años radiocarbónicos antes del presente (AP), mientras que se mantiene el tratamiento clásico de considerar a 10 ka como el límite entre Pleistoceno y Holoceno.

\section{RESULTADOS}

\section{Aspectos taxonómicos}

En la secuencia de TA1 se exhumaron restos referibles a roedores cricétidos, roedores caviomorfos y lagomorfos exóticos (Anexos 1 y 2). Seguidamente, se detallan los taxones registrados (Fig. 1), reseñándose en forma sucinta aspectos de sus estados sistemáticos -en el contexto geográfico del extremo austral de América del Sur- como así también sus principales rasgos diagnósticos y preferencias ambientales. Esto último tiene por objeto brindar un adecuado marco de información a los efectos del ulterior análisis de la significación paleoambiental.

\section{Roedores cricétidos}

Los representantes de la subfamilia Sigmodontinae constituyen el grupo taxonómico más diverso en TA1. En la secuencia del sitio se registran todas las especies -6 en total- que actualmente tienen poblaciones documentadas en Tierra del Fuego (véase Osgood, 1943; Massoia \& Chebez, 1993; Pardiñas et al. 2011b; Chebez et al. 2014; Fig. 2a), más una séptima sin menciones previas. Los taxones detectados son:

\section{Tribu Abrotrichini}

Abrothrix hirta (Thomas, 1895): muy tempranamente, Thomas (1908) describió una forma endémica, francei (hoy día sinónimo junior de hirta; véase Teta \& Pardiñas, 2014), sobre ejemplares de cercanías de El Porvenir, en el sector chileno de la isla. Colectas posteriores indican su presencia tanto en el sector norte (Estancia Sara; Massoia, 1983; citado como Abrothrix longipilis) como, plausiblemente, en el sur (Parque Nacional Tierra del Fuego, bahía Lapataia; Marconi, 1988; aunque podría tratarse de una confusión con Abrothrix lanosa, que en parte de su historia taxonómica fue considerado sinónimo junior de A. longipilis). Es la mayor especie de Abrothrix en Tierra del Fuego, caracterizada por mandíbulas alargadas y dientes de patrón oclusal abrotriquino, robustos $y$ elongados. En el continente, la especie presenta poblaciones tanto en el bosque como en la estepa (Osgood, 1943). Una revisión exhaustiva de los datos disponibles para Tierra del Fuego la indican como muy escasa, con la casi totalidad de las capturas restringidas a la porción norte de la isla, siempre en ambientes esteparios (Fig. 2b). 

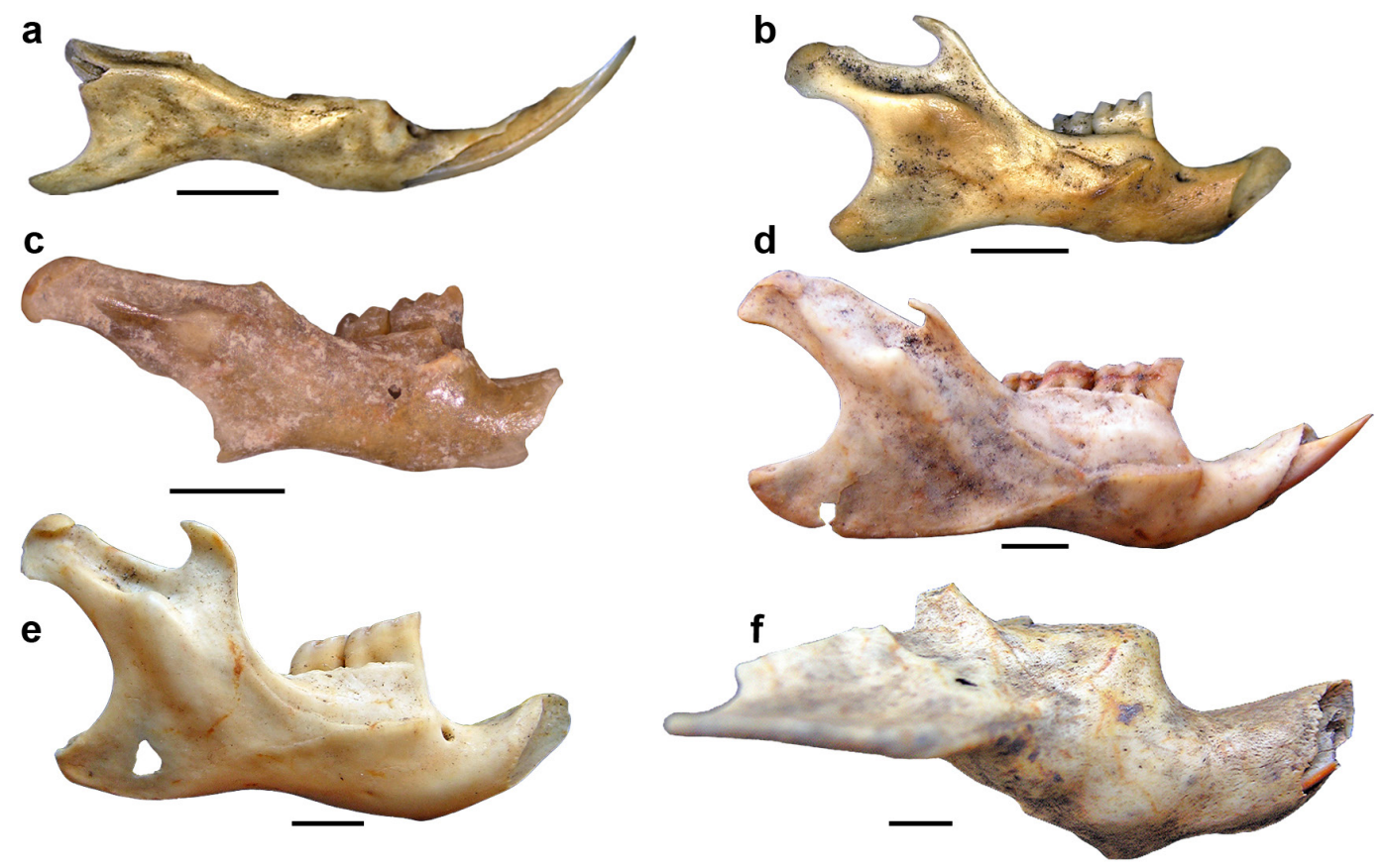

Fig. 1. Restos de roedores, hemimandíbulas derechas en vista labial, representativos de algunos taxones registrados en Tres Arroyos 1 (Tierra del Fuego, Chile): a, Abrothrix lanosa (capa II, cuadrícula D); b, Abrothrix olivacea (capa II, cuadrícula D); c, Eligmodontia cf. E. morgani (capa VI, nivel -170-175 cm, cuadrícula D); d, Reithrodon auritus (capa III superior, cuadrícula D); e, Euneomys chinchilloides (capa III superior, cuadrícula D); f, Ctenomys magellanicus (capa II, cuadrícula D). Escalas=2,5 mm.

Abrothrix lanosa (Thomas, 1897): con localidad típica en el archipiélago Madre de Dios (Chile; véase Feijoo et al. 2010), esta forma ha sido registrada en unas pocas localidades del continente y de las islas australes (e.g. Osgood, 1943; Feijoo et al. 2010; Guzmán Sandoval, 2010). Prima facie, aparece vinculada al bosque de Nothofagus y sectores colindantes, especialmente estepas arbustivas y graminosas en ambientes húmedos (Galliari \& Pardiñas, 1999). Sin embargo, se sabe muy poco de su distribución y preferencias ecológicas. En Tierra del Fuego es moderadamente frecuente sobre la costa sur, en matorrales de mata negra (Junellia tridens) y calafate (Berberis sp.) asociados directamente, o no, a sectores boscosos. Los registros más norteños conocidos en territorio insular alcanzan la latitud del lago Fagnano (Osgood, 1943; Feijoo et al. 2010), es decir, unos $130 \mathrm{~km}$ (en línea recta) al sur del emplazamiento de TA1 (Fig. 2c). A. lanosa resulta morfológicamente característico en el contexto de los sigmodontinos fueguinos, por sus mandíbulas notablemente gráciles y finas con molares muy pequeños (Feijoo et al. 2010; Fig. 1a).

Abrothrix olivacea (Waterhouse, 1837): con una extensa y complicada historia taxonómica, plagada de formas nominales -incluso acuñadas en áreas insulares australes- cuyos estatus están pendientes de contrastación (e.g. llanoi, hershkovitzi, canescens, xanthorhinus). Aquî se sigue el criterio de Pearson \& Smith (1999) al considerar un único taxón de amplia distribución en Patagonia continental e insular (véase, además, Chebez et al. 2014). Dicha hipótesis taxonómica debe ser adecuadamente puesta a prueba. A. olivacea es uno de los sigmodontinos hoy día más frecuentes en Tierra del Fuego (incluyendo las islas del Cabo de Hornos), incluso uno de los pocos que se captura en las áreas de bosque y turbera más densos y húmedos (Osgood, 1943; Pine et al. 1979; Patterson et al. 1984; U. Pardiñas, datos no publicados; Fig. 2d). En tal sentido, se destaca como un taxón de amplia labilidad ecológica y resistencia al impacto antrópico. Sus restos se 
distinguen de los otros representantes del género Abrothrix en Tierra del Fuego por sus mandíbulas cortas y comparativamente altas (Fig. 1b), con molares pequeños y de procíngulos comprimidos en sentido antero-posterior.

Tribu Euneomyini

Euneomys chinchilloides (Waterhouse, 1839): con una complicada historia nomenclatorial y taxonómica (véase Osgood, 1943), aún sin llegar a una total clarificación (véase Reise \& Gallardo, 1990; Pearson \& Christie, 1991; Braun \& Pardiñas, 2015; Ojeda et al. 2015), esta especie tiene su localidad típica, aunque de localización imprecisa, en el norte de Tierra del Fuego (Waterhouse, 1839). Al igual que Reithrodon, los restos de Euneomys se reconocen sin mayor problemática en función de sus molares de diseño sigmoide, con procíngulos de contorno redondeado, generalmente separados del resto de las estructuras oclusales, placas cigomáticas altas y estrechas de bordes paralelos e incisivos superiores con surco frontal único y mandíbulas con la rama ascendente bien vertical (Fig. 1e). Euneomys presenta una amplia distribución en Patagonia continental $e$ insular, alcanzando las islas del Cabo de Hornos (Olrog, 1950). Muestra una clara preferencia por los ambientes hostiles, peladales y pedregales muy expuestos, registrándose en estos microhábitats tanto en matrices de paisaje dominadas por bosques como por estepas, especialmente aquellas graminosas (Mann Fischer, 1978). En Tierra del Fuego se lo registra en todo el territorio (Osgood, 1943; Pine et al. 1979; Reise \& Venegas, 1987; Braun \& Pardiñas, 2015; Fig. 2e).

Tribu Oryzomyini

Oligoryzomys longicaudatus (Bennett, 1832): aquí se considera a $O$. magellanicus sinónimo junior de O. longicaudatus (contra Weksler \& Bonvicino, 2015; véase, además, Palma \& Rodríguez-Serrano, 2018). O. longicaudatus se distribuye en todo el territorio de Tierra del Fuego, pero resulta más abundante sobre el sector sur de la isla, en la franja boscosa y periboscosa, alcanzando también las islas del Cabo de Hornos (Osgood, 1943; Fig. 2f). La complejidad de los molares, por la presencia de mesolofos/lófidos bien desarrollados, hace de este taxón inconfundible en el contexto del conjunto fueguino.

Tribu Phyllotini

Eligmodontia cf. E. morgani Allen, 1901: esta especie presenta una amplia distribución patagónica, alcanzando la región del Parque Nacional Torres del Paine, Magallanes, Chile (Rau et al. 1978) y el extremo sur de Santa Cruz, en proximidades de monte Aymond (Pardiñas et al. 2009) y cabo Vírgenes (Pardiñas et al. 2011b). No existen poblaciones actuales documentadas en territorio insular austral (Osgood, 1943). Su hallazgo en TA1 es relevante y amerita una más profunda discusión (véase más adelante). Es un elemento típico de la estepa arbustiva, incluyendo ambientes con abundante suelo desnudo, roquedales, peladales y sectores litorales arenosos (Pearson, 1995; Mares et al. 2008). El conocimiento taxonómico de las poblaciones patagónicas australes de Eligmodontia es aún precario (Pardiñas et al. 2003; Lanzone et al. 2015). Estudios genéticos y morfológicos indican la existencia de una única especie, E. morgani, al sur de los $50^{\circ} \mathrm{S}$ (Lessa et al. 2010). Eligmodontia se caracteriza por molares pequeños, moderadamente braquiodontes, simples y una cresta masetérica que forma un reborde conspicuo en su extremo anterior (Fig. 1c).

\section{Tribu Reithrodontini}

Reithrodon auritus (Fischer, 1814): si bien sobre la base de ejemplares colectados tanto en Tierra del Fuego como en la región patagónica continental se han acuñado numerosos nombres (i.e. hatcheri, flammarum, obscurus, pachycephalus), ya Osgood (1943) propuso considerarlos sinónimos de R. auritus. Esta postura, que hasta el día de hoy no ha sido revisada críticamente (Pardiñas \& Galliari, 2001), se sigue en el presente trabajo (Pardiñas et al. 2015). Los restos de Reithrodon son fácilmente determinables, aun estando muy fragmentados, por una sumatoria única de rasgos: incisivos superiores con doble surco frontal, placa cigomática con proyección espinosa en su porción antero-dorsal, 
frontales estrechos de bordes paralelos, molares hipsodontes de coronas planas y laminados, rama mandibular alta con proceso coronoides proporcionalmente pequeño, sector interno del cóndilo mandibular bien excavado y fórmula radicular compleja (Pardiñas \& Galliari, 2001; Fig. 1d). R. auritus es un elemento característico de la estepa patagónica, especialmente abundante en sus sectores más graminosos, incluso hasta el linde del bosque (Pearson, 1988; Pardiñas \& Galliari, 2001). En Tierra del Fuego se lo registra desde la latitud del lago Fagnano hacia el norte (Osgood, 1943; Fig. 2g).

\section{Roedores caviomorfos}

El único género y especie de caviomorfo que presenta poblaciones vivientes en Tierra del Fuego es el tuco tuco Ctenomys magellanicus Bennett, 1836. Se han propuesto subespecies endémicas insulares para la misma, cuyos estatus y límites distribucionales deben estudiarse con más detalle (Fasanella et al. 2013; Bidau, 2015). La distribución fueguina de C. magellanicus se restringe al sector norte del territorio, tanto en estepas graminosas como arbustivas, con alguno que otro registro aislado para ambientes hoy día boscosos hasta la latitud del lago Fagnano (Osgood, 1943; Fig. 2h). Los restos de Ctenomys resultan inconfundibles en el contexto de los roedores de Tierra del Fuego (Santiago et al. 2016; Fig. 1f).

\section{Lagomorfos exóticos}

Oryctolagus cuniculus (Linnaeus, 1758), el conejo europeo común, fue introducido (ca. 1936) en Tierra del Fuego con fines peleteros y hoy día se encuentra asilvestrado en gran parte del territorio (e.g. Massoia \& Chebez, 1993; Chebez et al. 2014).

\section{Conjuntos de pequeños}

mamíferos y estratigrafía

Existe una correlación positiva y significativa entre los tamaños de las muestras (medidos en NISP craneano total; Tablas 1 y 2) y el número de especies, ya sea que se calcule por cuadrículas (A/B, r=0,808; D, r=0,828) o en la sumatoria total (TA1 [total roedores], $r=0,922$ ). Esto indica que la riqueza específica observada en TA1 es dependiente del tamaño de la muestra estudiado por capa, algo usual en muchos análisis de arqueofaunas. Más allá de este factor limitante, se observan diferencias importantes en la distribución estratigráfica de los taxones registrados, tanto en abundancias (Tablas 1 y 2) como en presencia/ ausencia (Tabla 3). Seguidamente se efectúa un análisis comentado, desde los niveles inferiores del sitio hasta los superiores.

Capa VI: una muestra pequeña ( $\mathrm{NISP}=34$, $\mathrm{MNI}=12$, exclusivamente cuadrícula $\mathrm{D})$ revela 3 taxones, con amplia dominancia de $E$.

Tabla 1. NISP (restos craneanos) y NISP\% por capa y taxón (orden alfabético) para las muestras de micromamíferos de la cuadrícula A/B de Tres Arroyos 1 (Tierra del Fuego, Chile).

Taxones y restos considerados intrusivos han sido excluidos.

\begin{tabular}{lcccccccccc}
\hline & \multicolumn{2}{c}{ I } & \multicolumn{2}{c}{ II } & \multicolumn{2}{c}{ III } & \multicolumn{2}{c}{ IV } & \multicolumn{2}{c}{ Va } \\
Especie & NISP & $\%$ & NISP & $\%$ & NISP & $\%$ & NISP & $\%$ & NISP & $\%$ \\
\hline A. hirta & & & & & 4 & 0,59 & 1 & 0,11 & & \\
A. lanosa & & & & & 6 & 0,88 & 2 & 0,22 & & \\
A. olivacea & & & & & 11 & 1,62 & 14 & 1,52 & 3 & 1,75 \\
C. magellanicus & 15 & 24,20 & 26 & 29,20 & 121 & 17,80 & 111 & 12,10 & 21 & 12,30 \\
E. chinchilloides & 12 & 19,40 & 14 & 15,70 & 170 & 25,10 & 596 & 64,80 & 141 & 82,50 \\
O. longicaudatus & & & 1 & 1,12 & 2 & 0,29 & 1 & 0,11 & & \\
R. auritus & 35 & 56,50 & 48 & 53,90 & 364 & 53,70 & 195 & 21,20 & 6 & 3,51 \\
\hline Total & 62 & & 89 & & 678 & & 920 & & 171 & \\
Riqueza (S) & & 3 & & 4 & & 7 & & 7 & & 4 \\
\hline
\end{tabular}




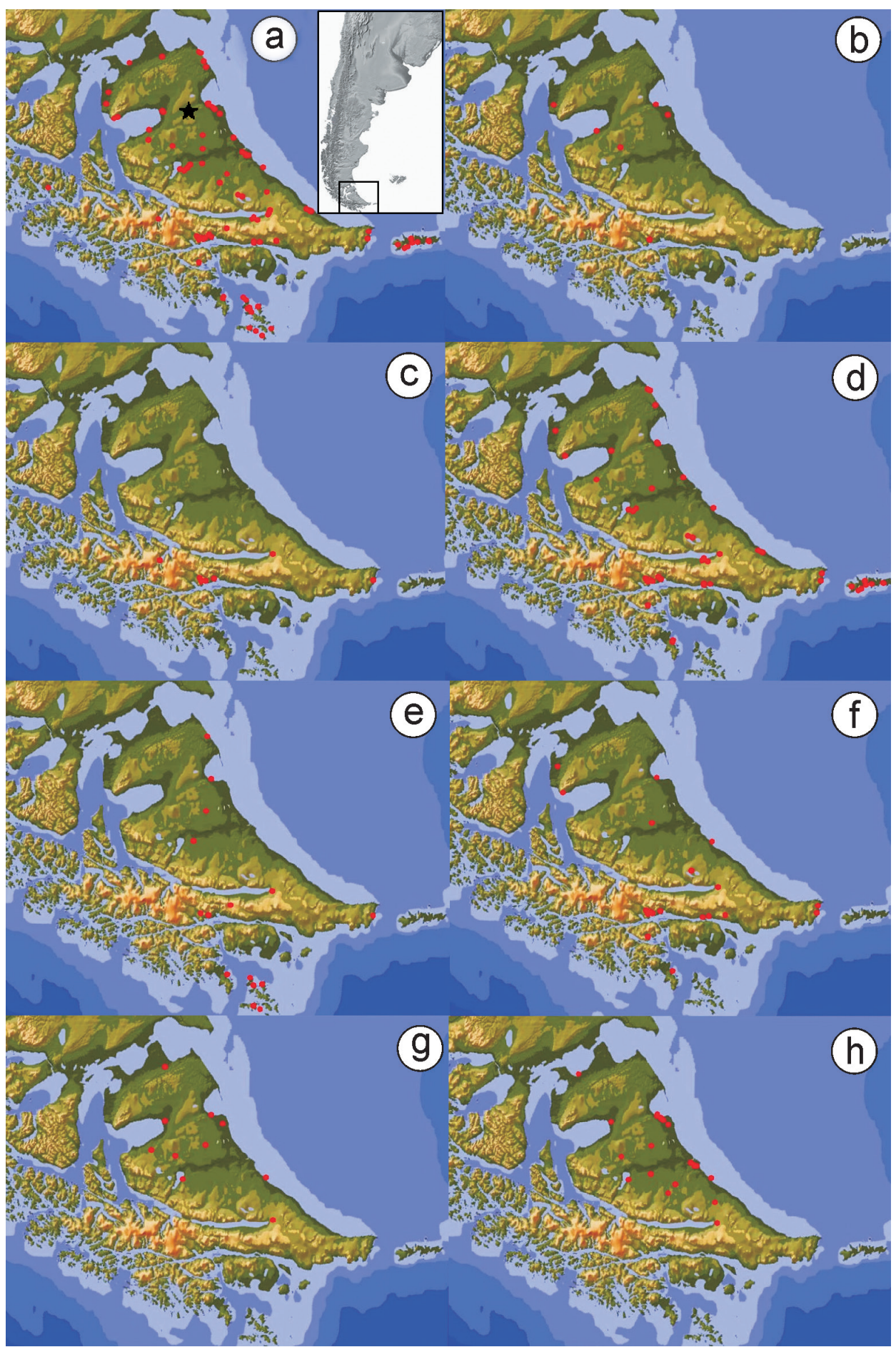

Fig. 2. Distribuciones geográficas (puntos de registro, en rojo) de los roedores fueguinos:

a, todos los registros disponibles para roedores (la estrella negra señala la ubicación del sitio Tres Arroyos 1); b, Abrothrix hirta; c, Abrothrix lanosa; d, Abrothrix olivacea; e, Euneomys chinchilloides; f, Oligoryzomys longicaudatus; g, Reithrodon auritus; h, Ctenomys magellanicus. Datos compilados de varias fuentes, incluyendo literatura y revisión de colecciones. 
Tabla 2. NISP (restos craneanos) y NISP\% por capa y taxón (orden alfabético) para las muestras de micromamíferos de la cuadrícula D de Tres Arroyos 1 (Tierra del Fuego, Chile).

Se excluyeron los taxones y restos considerados intrusivos.

\begin{tabular}{|c|c|c|c|c|c|c|c|c|c|c|c|c|c|c|}
\hline \multirow[b]{2}{*}{ Especie } & \multicolumn{2}{|c|}{ II } & \multicolumn{2}{|c|}{ III superior } & \multicolumn{2}{|c|}{$\mathrm{IVa}$} & \multicolumn{2}{|c|}{$\mathrm{IVb}$} & \multicolumn{2}{|c|}{$\mathrm{Va}$} & \multicolumn{2}{|c|}{$\mathrm{Vb}$} & \multicolumn{2}{|c|}{$\mathrm{VI}$} \\
\hline & NISP & $\%$ & NISP & $\%$ & NISP & $\%$ & NISP & $\%$ & NISP & $\%$ & NISP & $\%$ & NISP & $\%$ \\
\hline A. hirta & & & & & & & & & 1 & 0,19 & & & & \\
\hline A. lanosa & 1 & 0,21 & & & & & & & & & & & & \\
\hline A. olivacea & 1 & 0,21 & 1 & 0,21 & 1 & 0,17 & & & 2 & 0,37 & & & & \\
\hline C. magellanicus & 130 & 26,69 & 128 & 26,83 & 143 & 24,24 & 57 & 27,80 & 102 & 19,07 & 36 & 46,15 & 3 & 8,82 \\
\hline Eligmodontia sp. & & & & & & & & & & & & & 3 & 8,82 \\
\hline E. chinchilloides & 107 & 21,97 & 105 & 22,01 & 199 & 33,73 & 91 & 44,39 & 414 & 77,38 & 41 & 52,56 & 28 & 82,35 \\
\hline R. auritus & 248 & 50,92 & 243 & 50,94 & 247 & 41,86 & 57 & 27,80 & 16 & 2,99 & 1 & 1,28 & & \\
\hline Total & 487 & & 477 & & 590 & & 205 & & 535 & & 78 & & 34 & \\
\hline Riqueza (S) & & 5 & & 4 & & 4 & & 3 & & 5 & & 3 & & 3 \\
\hline
\end{tabular}

Tabla 3. Distribución estratigráfica de los micromamíferos registrados en la secuencia de Tres Arroyos 1 (Tierra del Fuego, Chile). Datos combinados cuadrículas A/B y D; taxones y restos considerados intrusivos han sido excluidos; unidades estratigráficas por antigüedad creciente de derecha a izquierda.

\begin{tabular}{|c|c|c|c|c|c|}
\hline & \multicolumn{2}{|c|}{ Holoceno tardío } & \multicolumn{3}{|c|}{ Pleistoceno tardío } \\
\hline & I & $\begin{array}{lll}\text { II } & \text { III } & \text { IV }\end{array}$ & $\mathrm{Va}$ & $\mathrm{Vb}$ & VI \\
\hline \multicolumn{6}{|l|}{ Abrothrix hirta } \\
\hline \multicolumn{6}{|l|}{ Abrothrix lanosa } \\
\hline \multicolumn{6}{|l|}{ Abrothrix olivacea } \\
\hline \multicolumn{6}{|c|}{ Ctenomys magellanicus } \\
\hline \multicolumn{6}{|l|}{ Eligmodontia sp. } \\
\hline \multicolumn{6}{|c|}{ Euneomys chinchilloides } \\
\hline \multicolumn{6}{|l|}{$\begin{array}{l}\text { Oligoryzomys } \\
\text { longicaudatus }\end{array}$} \\
\hline Reithrodon auritus & & & & & \\
\hline
\end{tabular}

chinchilloides y escasos restos de C. magellanicus. Se verifica, asimismo, la única ocurrencia en toda la secuencia de Eligmodontia cf. E. morgani, un sigmodontino ausente en la mastofauna actual de la isla. R. auritus, dominante en los niveles superiores de TA1, está ausente.

Capa Vb: con un tamaño de muestra algo mayor que en la capa subyacente (NISP=78, $\mathrm{MNI}=38$, exclusivamente cuadrícula $\mathrm{D}$ ), se registran 3 especies, codominando $C$. magellanicus y E. chinchilloides. Los restos de R. auritus son, en función de su estado, mayoritariamente intrusivos (Anexo 2); solo un hemimaxilar derecho resulta, juzgado por su estado de conservación, presumiblemente autóctono. Este material, recuperado en el rango estratigráfico -100 a -108 $\mathrm{cm}$ de profundidad representa, si se acepta como contemporáneo del sedimento portador, el primer registro de $R$. auritus para la secuencia de TA1.

Capa Va: con tamaños de muestra importantes (NISP $=171, \quad \mathrm{MNI}=62$ [cuadrícula $\mathrm{A} / \mathrm{B}$ ], $\mathrm{NISP}=535, \quad \mathrm{MNI}=241 \quad$ [cuadrícula D]), puede considerarse una muestra representativa del conjunto de micromamíferos hacia el Pleistoceno 
más tardío. La riqueza de especies trepa a 5 , con la incorporación de 2 de Abrothrix a las reseñadas para capa $\mathrm{Vb}$. Con respecto a $R$. auritus, de los 95 restos registrados (Anexos 1 y 2), en su mayoría son claramente intrusivos. Unos pocos (Tablas 1 y 2) se consideran como autóctonos. Más allá de esto resulta clara la infrecuencia de este roedor con respecto a $E$. chinchilloides, que en esta capa alcanza un NISP=555 (ambas cuadrículas combinadas).

Capa IV: con la mayor muestra de toda la secuencia (NISP total=1.715), en un conjunto de 7 especies registradas se verifica la primera aparición de $A$. lanosa y $O$. longicaudatus. $R$. auritus incrementa su frecuencia desde la parte inferior de esta capa (IVb) hacia la superior (IVa), donde prácticamente se equipara en relación con E. chinchilloides.

Capa III: con algo más de mil restos estudiados, la riqueza es igual a aquella de capa IV. $R$. auritus pasa a ser el sigmodontino dominante, supremacía que ya no se perderá en los niveles superiores.

Capa II: con 6 especies registradas, la ausencia de $A$. hirta no puede desvincularse, al menos teóricamente, de su menor tamaño de la muestra (NISP total=576). Este conjunto, cronológicamente previo al impacto antrópico por ganadería extensiva, puede considerarse un buen parámetro comparativo para la reconstrucción paleoambiental.

Capa I: el pequeño tamaño de la muestra (NISP=62, $\mathrm{MNI}=21$, exclusivamente cuadrícula $\mathrm{A} / \mathrm{B})$ no permite mayores inferencias, quedando reducida la riqueza a los 3 taxones dominantes en toda la secuencia de TA1.

Comparando los segmentos temporales involucrados, esto es, Pleistoceno tardío vs. Holoceno tardío pre-ovino, se observan diferencias destacables entre los conjuntos (Tabla 3). Aquel del Pleistoceno tardío (ca. 10-12,5 ka AP) se caracteriza por una riqueza baja, con una dominancia absoluta de E. chinchilloides y la aparición tardía de A. hirta, A. olivacea y $R$. auritus. Además, se registra un taxón hoy día extinto (Eligmodontia). Durante el Holoceno tardío (ca. 0,13-1,3 ka AP) se enriquece considerablemente el conjunto de especies, con la adición de O. longicaudatus y A. lanosa. Además, $R$. auritus pasa a ser dominante con respecto a
E. chinchilloides. Tentativamente, estos cambios de riqueza y abundancias entre los dos segmentos temporales podrían estar vinculados con factores climático-ambientales.

\section{Aspectos tafonómicos básicos}

Sin dudas la muestra de roedores de TA1 amerita un análisis tafonómico exhaustivo. Las observaciones que aquí se brindan deben considerarse como básicas y en ningún sentido agotan el tema. Pretenden, en última instancia, una primera aproximación a dos cuestiones fundamentales: el proceso de incorporación de los roedores al yacimiento y el carácter primario o secundario de su asociación con el sedimento portador. Como atributos básicos para atacar ambas cuestiones se destacan el estado superficial de los restos y las abundancias de elementos esqueletarios.

Los restos correspondientes a las capas I a IV presentan, en rasgos generales, superficies limpias sin presencia de concreciones, nula o muy escasa mineralización (Fig. 3), orificios muchas veces rellenos por sedimento terroso fácilmente removible sin acción mecánica agresiva, elementos dentarios lábilmente unidos, marcas de raíces superficiales y abundantes (Fig. 3a), color variable pero en la gama del ocre a marrón oscuro, en una buena proporción con manchas puntiformes de manganeso (Fig. 3b). En claro contraste, aquellos de capa Va son frágiles, de color ambarino o amarillento (Fig. 4a), generalmente limpios y no presentan aspecto "fresco" (determinado por alta porosidad, nula mineralización y/o existencia de sedimento adherido). Los de capa Vb y VI están básicamente recubiertos de concreciones -ya fueran oscuras tipo manganesíferas o formadas por partículas tamaño arena- tenaces y los molariformes e incisivos más sólidamente vinculados a sus alvéolos, sugiriendo algún tipo de sustancia cementante (Fig. 4b).

Con estos elementos de juicio, logrados mediante la evaluación general de los conjuntos, varios materiales de la capa $\mathrm{V}$ se pudieron identificar como alóctonos. Se trata, en su mayoría, de restos de $R$. auritus, pero también los hay de $C$. magellanicus y E. chinchilloides (Anexos 1 y 2). Por su parte, los restos de O. cuniculus, presentes 

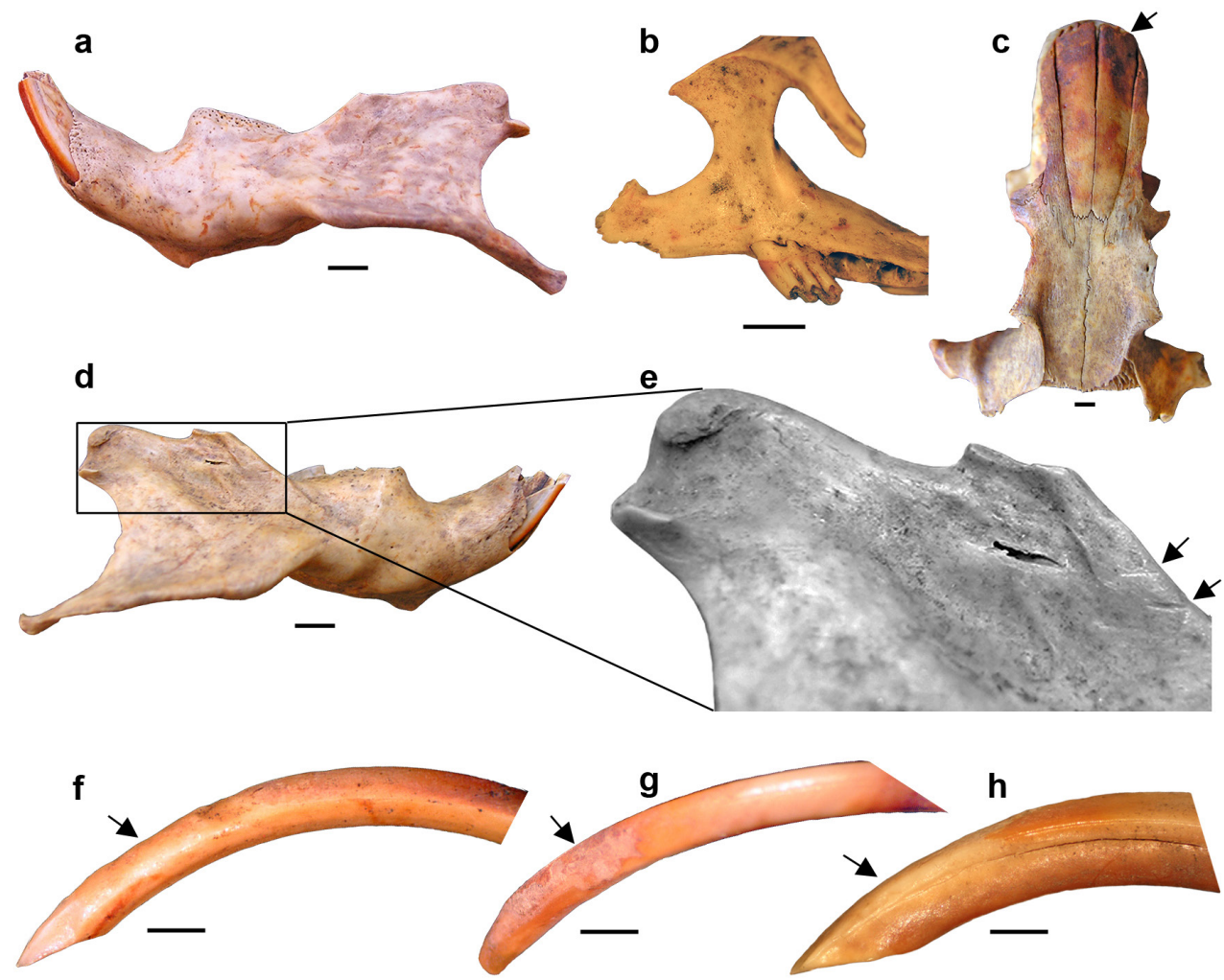

Fig. 3. Algunos ejemplos de los atributos tafonómicos identificados en las capas del Holoceno tardío del sitio arqueológico Tres Arroyos 1 (Tierra del Fuego, Chile): a, hemimandíbula izquierda de Ctenomys magellanicus con marcas de raíces, vista labial (capa III superior); b, hemimaxilar izquierdo de Reithrodon auritus con impresiones por óxido de manganeso, vista labial (capa IVb); c, fragmento de cráneo de C. magellanicus con termoalteración leve en su parte anterior (señalada por una flecha), vista dorsal (capa III superior); d, hemimandíbula derecha de C. magellanicus con marcas de corte en la base del proceso coronoides y detalle magnificado de las mismas (e), vista labial (capa II); f, incisivo inferior de cricétido con marcas de corrosión ligera (capa II); g, incisivo inferior de cricétido con marcas de corrosión ligera (capa III superior); h, incisivo inferior de $C$. magellanicus con marcas de corrosión ligera (capa III superior). Escalas: a-d=2,5 mm; $\mathrm{f}-\mathrm{h}=1 \mathrm{~mm}$.

en varias capas, siempre en su estado superficial mostraron un claro aspecto "fresco"; en todos los casos se trata de individuos juveniles.

Las muestras de las capas I a IV se caracterizan por una proporción importante (>50\%) de cráneos bien preservados. Por ejemplo, los cráneos de C. magellanicus aparecen completos en su porción anterior, en muchos casos conservando los nasales $y$ la separación del posterocráneo ha seguido la sutura coronal (Fig. 3c). Muchas veces, las regiones craneanas posteriores, involucrando occipitales y bullas timpánicas, también integran las muestras (no fueron cuantificadas). Del mismo modo, las hemimandíbulas conservan típicamente los procesos condiloideos y angulares, no así, en general, los coronoides (Fig. 3a). Las muestras de las capas V y VI están integradas por restos que acusan una mayor fragmentación. En estas capas, la presencia de cráneos de C. magellanicus es totalmente esporádica; las hemimandíbulas, en su mayoría, aparecen reducidas a sus porciones de la rama horizontal (Fig. 4a, 4c).

Una evaluación del detalle por capa y cuadrícula (Anexos 1 y 2) muestra que las capas superiores (I a IV) se caracterizan por mayores proporciones de cráneos y paladares de $C$. magellanicus, en contraste con las capas inferiores (V-VI). Este comportamiento queda en evidencia con la aplicación del índice de supervivencia (Is, sensu Pardiñas, 1999b; Tabla 
4). Los valores de Is para C. magellanicus aumentan hacia los niveles inferiores. Aquellos calculados para $R$. auritus son notablemente estables y cercanos a 1 , indicando proporciones hemimaxilar/hemimandíbula equiparables. Por su parte, los de E. chinchilloides muestran una clara desproporción en favor de las hemimandíbulas, arrojando valores de Is sensiblemente más elevados.

La mayor parte de los restos de C. magellanicus exhibió los incisivos -cuando éstos se conservaron en sus alvéolos- distalmente quebrados (Fig. 3d). Por ejemplo, para una muestra de capa II (cuadrícula D), sobre 44 hemimandíbulas derechas, 30 conservando los incisivos, solo 2 estaban apicalmente completos; en la misma muestra, sobre 21 fragmentos anteriores de cráneo, de los 9 que poseían incisivos todos estaban quebrados. En una muestra de la capa III superior (cuadrícula D), sobre 40 hemimandíbulas derechas y 22 conservando el incisivo, solo 3 estaban enteros distalmente. Se observó que la

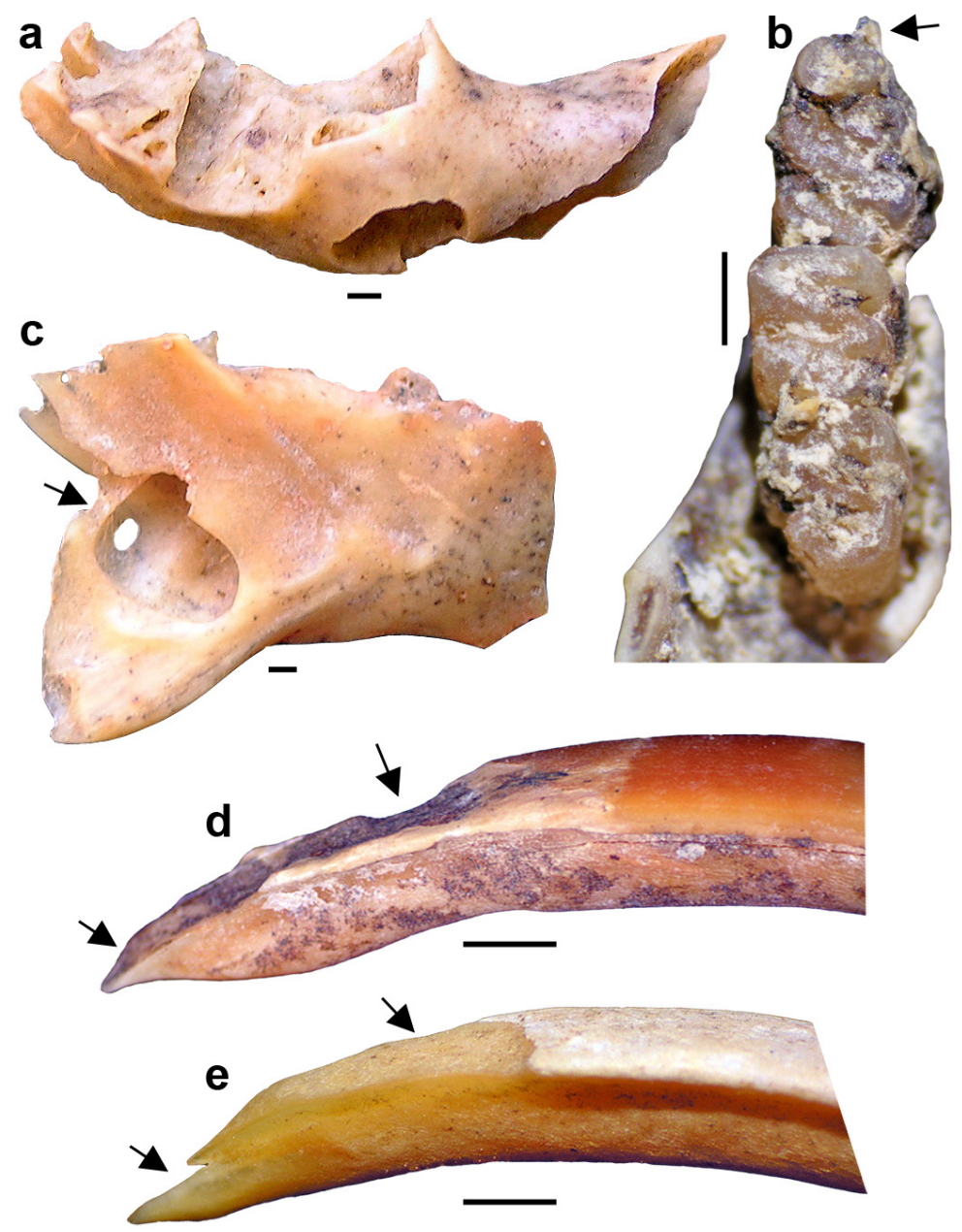

Fig. 4. Ejemplos de los atributos tafonómicos identificados en las capas del Pleistoceno tardío del sitio arqueológico Tres Arroyos 1 (Tierra del Fuego, Chile): a, hemimandíbula derecha muy fragmentada de Ctenomys magellanicus, vista labial (capa Va); b, serie molar inferior izquierda de Euneomys chinchilloides con concreciones minerales, vista oclusal (capa Vb); c, fragmento de la rama horizontal de hemimandíbula derecha de $C$. magellanicus con evidencias de corrosión digestiva, vista labial (capa Va); d, incisivo inferior de C. magellanicus con marcas de corrosión moderada (capa Va); e, incisivo inferior de C. magellanicus con marcas de corrosión fuerte (capa Va). Escalas: $1 \mathrm{~mm}$. 
Tabla 4. Valores del índice de supervivencia por capa y taxón para muestras seleccionadas por NISP total de la secuencia de Tres Arroyos 1 (Tierra del Fuego, Chile).

\begin{tabular}{lccc}
\hline Cuadrícula y capa & C. magellanicus & R. auritus & E. chinchilloides \\
\hline A/B, capa I & 1,25 & 1,19 & 11,00 \\
A/B, capa II & 1,36 & 1,29 & 3,67 \\
A/B, capa III & 1,01 & 0,99 & 1,95 \\
A/B, capa IV & 1,65 & 0,96 & 2,01 \\
D, capa II & 1,27 & 1,19 & 3,40 \\
D, capa III superior & 1,29 & 0,88 & 2,00 \\
D, capa IVa & 1,63 & 0,80 & 6,18 \\
D, capa IVb & 2,00 & 0,89 & 8,71 \\
D, capa Va & 3,76 & - & 6,18 \\
D, capa Vb & 2,89 & - & - \\
\hline
\end{tabular}

fragmentación también afectó masivamente a los molariformes, en muchos casos mediante una estría longitudinal separando al diente en dos porciones de esmalte-dentina y en otros, a partir de esta estría, otras radiales menores.

La mayor parte de los restos de $C$. magellanicus de los niveles holocénicos corresponde a ejemplares adultos, al menos a juzgar por sus tamaños comparados con materiales de referencia de peso conocido. Se trata de individuos que alcanzarían los 450-500 gramos. Hay restos asignables a juveniles o subadultos, pero en proporciones minoritarias.

Prácticamente, no se detectaron materiales termoalterados. Los pocos casos corresponden a restos de C. magellanicus de los niveles holocénicos (Fig. 3c). En el mismo período, un único resto parece comportar marcas asignables a corte; se trata de una hemimandíbula de $C$. magellanicus proveniente de la capa II, donde se observan finas estrías poco profundas sobre el sector basal del proceso coronoides (Fig. 3d, 3e).

El examen de las marcas de corrosión digestiva en muestras seleccionadas de incisivos aislados (capas II, III y Va) permitió una aproximación cuantitativa (Tabla 5). Estos incisivos, seguramente en su gran mayoría, corresponden a piezas dentarias liberadas de los maxilares y mandíbulas durante las etapas de excavación, almacenado, separación, transporte y proceso de estudio del material (etapa final de la historia tafonómica de los agregados, cf. Andrews, 1990). Estos elementos dentarios pertenecen a Ctenomys, Euneomys y Reithrodon. La mayor

Tabla 5. Marcas de corrosión en muestras de incisivos de roedores de Tres Arroyos 1 (Tierra del Fuego, Chile).

\begin{tabular}{lccccccc}
\hline \multirow{2}{*}{ Grados de digestión } & \multicolumn{2}{c}{ Ligera } & \multicolumn{2}{c}{ Moderada } & \multicolumn{2}{c}{ Fuerte } \\
& NISP & Ctenomys & Otros & Ctenomys & Otros & Ctenomys & Otros \\
\hline Capa II & 187 & 1 & 10 & 0 & 0 & 2 & 1 \\
Capa III & 217 & 0 & 5 & 2 & 1 & 1 & 0 \\
Capa Va & 211 & 1 & 20 & 1 & 0 & 6 & 0 \\
Capa II, \% corrosión & & 0,53 & 5,35 & 0 & 0 & 1,07 & 0,53 \\
Capa III, \% corrosión & & 0 & 2,30 & 0,92 & 0,46 & 0,46 & 0 \\
Capa Va, \% corrosión & & 0,47 & 9,48 & 0,47 & 0 & 2,84 & 0 \\
\hline
\end{tabular}


Tabla 6. Abundancias relativas (sobre NISP) de elementos esqueletarios en muestras de micromamíferos de Tres Arroyos 1 (Tierra del Fuego, Chile).

\begin{tabular}{|c|c|c|c|c|c|c|c|c|c|}
\hline & \multicolumn{5}{|c|}{ Cuadrícula A/B } & \multicolumn{4}{|c|}{ Cuadrícula D } \\
\hline & I & II & III & IV & V & Va [sup] & Va [inf] & Vb [sup] & Vb [inf] \\
\hline Hemimandíbula & 27,01 & 24,66 & 16,88 & 18,55 & 15,15 & 2,90 & 5,34 & 2,86 & 4,44 \\
\hline Hemimaxilar & 15,33 & 11,21 & 6,56 & 10,22 & 9,13 & 0,41 & 1,48 & 0,82 & 0,00 \\
\hline Escápula & 0,00 & 1,35 & 2,62 & 2,74 & 2,09 & 0,83 & 0,59 & 2,45 & 0,00 \\
\hline Húmero & 14,60 & 6,28 & 13,77 & 14,33 & 17,50 & 29,88 & 23,44 & 27,35 & 23,70 \\
\hline Radio & 2,19 & 0,90 & 6,98 & 3,97 & 3,98 & 7,47 & 5,93 & 7,76 & 3,70 \\
\hline Cúbito & 3,65 & 3,59 & 6,32 & 5,41 & 6,02 & 9,96 & 10,98 & 17,55 & 12,59 \\
\hline Pelvis & 7,30 & 11,66 & 12,34 & 10,64 & 10,10 & 3,73 & 4,75 & 2,45 & 2,96 \\
\hline Fémur & 17,52 & 15,70 & 16,16 & 19,53 & 20,71 & 23,65 & 27,00 & 16,33 & 29,63 \\
\hline Tibia & 12,41 & 24,66 & 18,37 & 14,61 & 15,31 & 21,16 & 20,47 & 22,45 & 22,96 \\
\hline NISP & 137 & 223 & 1677 & 2847 & 1960 & 241 & 337 & 245 & 135 \\
\hline
\end{tabular}

parte de los ejemplares no muestra señales de corrosión. Las capas II y III exhiben baja proporción de incisivos con marcas de digestión (Tabla 5), mayoritariamente del grado ligera, donde solo el esmalte se ve afectado (Fig. 3f-3h). La capa Va muestra el mayor porcentaje general de corrosión, con un $13,2 \%$ sobre NISP total (211 incisivos); $\mathrm{n}$ las categorías ligera, moderada (con remoción del esmalte y dentina ondulada, Fig. 4d) y fuerte (que involucra la eliminación de buena parte del esmalte y la deformación de la dentina, véase Fernández et al. 2017b), particularmente sobre Ctenomys (Fig. 4e).

También se han detectado restos craneanos -principalmente hemimandíbulas- con evidencias de digestión ósea. Pertenecen a Ctenomys en su totalidad y se caracterizan por haber quedado reducidos a las porciones más resistentes de la rama horizontal (Fig. 4a, 4c), mostrando perforaciones en la base de los alvéolos con total remoción ósea y superficies suaves ("pulidas"). Son característicos de los niveles pleistocénicos de TA1.

Las abundancias relativas de elementos esqueletarios (Tabla 6, Fig. 5) mostraron valores similares para las capas I a Va de la cuadrícula A/B, con máximos en hemimandíbula, húmero, fémur y tibia. Por su parte, aquellas muestras de la cuadrícula $\mathrm{D}$-exclusivamente capa $\mathrm{V}$ (Pleistoceno tardío)- también expresaron valores similares entre sí, con máximos en húmero, cúbito, fémur y tibia. Es interesante destacar un comportamiento diferencial en las abundancias relativas entre ambos bloques temporales, situación que puede deberse a múltiples factores (diferencias metodológicas durante la excavación y recuperación del material, preservación diferencial, diferentes agentes tafonómicos según los sectores del sitio arqueológico). Si bien los valores de abundancia relativa son bajos, algo frecuente en conjuntos arqueológicos (Fernández, 2012), los patrones de los elementos esqueletarios registrados en las capas asociadas al Holoceno tardío coinciden con aquellos observados en muestras actuales generadas por aves rapaces nocturnas (estrigiformes; Fig. 5). En la capa Va se observa un patrón inverso en pelvis, propio de los conjuntos depositados por las aves rapaces diurnas y de los mamíferos carnivoros (Fig. 5).

Las marcas atribuibles a raíces caracterizan los restos de los niveles superiores de TA1, especialmente aquellos de Ctenomys. Generalmente, son cortas y superficiales, perceptibles por diferencias de coloración entre la porción ósea afectada y aquella remanente (Fig. 3a). Estas marcas postdepositacionales pueden ocultar los atributos inherentes a los agentes que acumularon los restos (e.g. Andrews, 1990; Fernández et al. 2019). 
Fig. 5. Abundancia relativa de partes esqueletarias en las muestras de micromamíferos del sitio arqueológico Tres Arroyos 1 (Tierra del Fuego, Chile): capas I, II, III y IV (Holoceno tardío) y capas V, $\mathrm{Va}, \mathrm{Vb}$ (Pleistoceno tardío). Se compara con los promedios para aves rapaces nocturnas (Strigiformes), rapaces diurnas (Accipitriformes y Falconiformes) y mamíferos carnívoros (Felidae, Canidae, Mephitidae y

Mustelidae), conocidos para América del Sur (sobre datos de Montalvo \& Fernández, 2019).

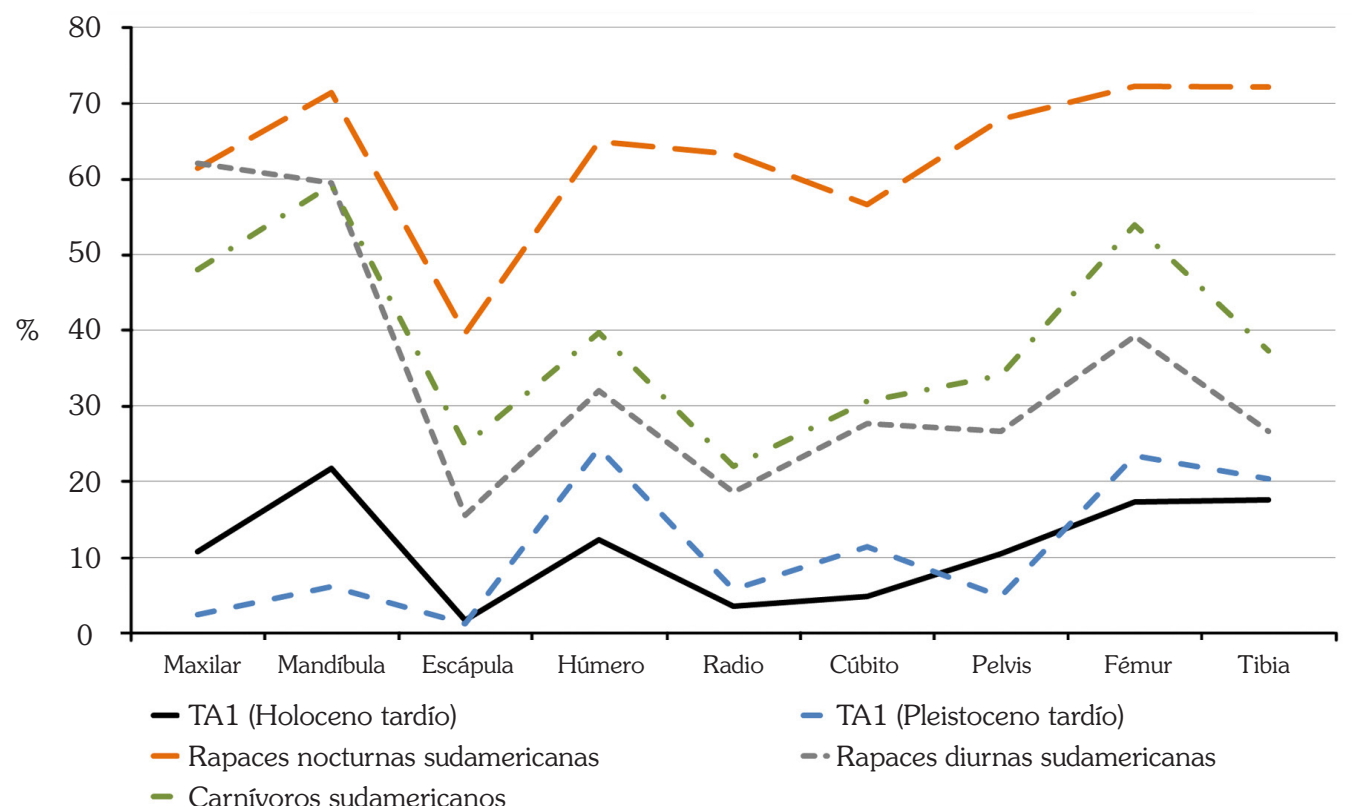

\section{DISCUSIÓN}

\section{Procesos de incorporación de micromamíferos}

La muestra de roedores de TA1 sugiere la participación de diferentes procesos de agregación. Más aún, es factible argumentar diferencias según los taxones considerados y según las capas.

El caso de Ctenomys magellanicus resulta un buen ejemplo de lo expresado (Tabla 7). La presencia de un resto con marca de cortes y de algún ejemplar termoalterado indica ingresos por causas antrópicas. El perfil etario, dominado por ejemplares adultos, sugiere una selección a favor de presas de mayor tamaño (aspecto compartido entre mamíferos carniceros y una potencial depredación por grupos humanos).

La elevada frecuencia de fracturas en incisivos y molariformes y la desarticulación del sector occipital (no traumático) revelan, por su parte, un tiempo considerable de permanencia de los restos expuestos al intemperismo previo a su enterramiento (cf. estadios 2 y 3 de meteorización de Andrews, 1990). Cuánto fue este tiempo es imposible conjeturar sin experimentos locales y/o regionales controlados.
Todas estas evidencias también excluyen un proceso mayoritario de incorporación natural por causas etoecológicas (cf. Pardiñas, 1999b; Tabla 7).

Las curvas de abundancias esqueletarias relativas para las capas I a IV exhiben similitud con aquellas obtenidas a partir del análisis de conjuntos generados por aves rapaces nocturnas (Fig. 5). Esto se traduce en máximos en hemimandíbulas, hemimaxilares, húmeros, fémures y tibias y mínimos en escápulas, radios, cúbitos y pelvis. Sin embargo, las frecuencias esqueletarias son indicadores débiles, al menos en muestras de micromamíferos, para la inferencia tafonómica de detalle (cf. Saavedra \& Simonetti, 1998).

Se han detectado claros ejemplos de corrosión plausiblemente referibles a la acción de ácidos digestivos sobre el material óseo y dentario (cf. Andrews, 1990; Fernández-Jalvo \& Andrews, 1992; Fernández et al. 2017b; Fernández \& Montalvo, 2019). La mayor parte de los casos de digestión fuerte se concentra sobre restos de Ctenomys.

Globalmente, los restos de micromamíferos de los niveles pleistocénicos de TA1 sugieren un origen mayoritario en la actividad depredadora 
Tabla 7. Contrastación de agentes tafonómicos (tipificados según Pardiñas, 1999b) y atributos observados en las muestras de restos de Ctenomys magellanicus del sitio Tres Arroyos 1 (Tierra del Fuego, Chile); no se consideran rasgos obvios, como actividad y tamaño.

\begin{tabular}{|c|c|c|c|c|}
\hline Agente tafonómico/atributo & $\begin{array}{c}\text { Capa } \\
\text { II }\end{array}$ & $\begin{array}{c}\text { Capa } \\
\text { III }\end{array}$ & $\begin{array}{c}\text { Capa } \\
\text { IV }\end{array}$ & $\begin{array}{c}\text { Capas } \\
\text { V-VI }\end{array}$ \\
\hline \multicolumn{5}{|l|}{ Mamíferos carniceros } \\
\hline -Fragmentación elevada & no & no & no & sí \\
\hline -Digestión severa & no & no & no & Sí \\
\hline -Selección etaria (adultos) & sí & sí & sí & sí \\
\hline \multicolumn{5}{|l|}{ Rapaces nocturnas } \\
\hline -Baja fragmentación & sí & sí & sí & no \\
\hline -Digestión leve a moderada & sí & sí & sí & no \\
\hline -Selección etaria (juveniles) & no & no & no & no \\
\hline -Desarticulación craneana & sí & sí & sí & $\dot{2} ?$ \\
\hline \multicolumn{5}{|l|}{ Consumo antrópico } \\
\hline -Marcas de corte & sí & no & no & no \\
\hline -Termoalteración & no & sí & no & no \\
\hline -Selección etaria (adultos) & sí & sí & sí & sí \\
\hline \multicolumn{5}{|c|}{ Natural por causas eto-ecológicas } \\
\hline -Baja fragmentación & sí & sí & sí & no \\
\hline -Digestión nula & sí & sí & sí & no \\
\hline -Sin selección etaria & no & no & no & no \\
\hline -Individuos articulados & $\dot{c} ?$ & $\dot{c} ?$ & $\dot{c} ?$ & $\dot{\imath} ?$ \\
\hline
\end{tabular}

de mamíferos carniceros. Permiten y robustecen esta hipótesis atributos relevados tales como elevada fragmentación, frecuencia de incisivos con evidencias de digestión fuerte y baja abundancia relativa de restos craneanos (cf. muestras cuadrícula D). En forma concomitante, en TA1 se han registrado restos de varios mamíferos potencialmente depredadores de roedores, incluyendo desde pequeños a grandes félidos y cánidos (véase la discusión en Martin, 2013).

Las muestras de niveles holocénicos de TA1 sugieren, como agente tafonómico principal, aves rapaces nocturnas (i.e. búhos y lechuzas). La baja proporción de marcas de corrosión de tipo ligera inclina la génesis hacia Tyto alba, una lechuza cuyos agregados aparecen poco modificados por procesos digestivos (Andrews,
1990; Saavedra \& Simonetti, 1998; Pardiñas, 1999b; Fernández et al. 2017b; Montalvo \& Fernández, 2019). La acción antrópica verificada en los niveles superiores de TA1 es coherente con hallazgos previos en la isla durante el Holoceno tardío, en un contexto de economías diversificadas de los cazadores recolectores, práctica que continuó hasta principios del siglo pasado (e.g. Santiago et al. 2016; Fernández et al. 2017a y literatura citada en estos trabajos). Resulta intrigante -en el contexto de esta hipótesis genética- la selección etaria observada en Ctenomys. Sin embargo, aunque hay pocos ejemplos publicados, se ha observado repetidamente un comportamiento trófico en $T$. alba que consiste en la partición de las presas mayores y el consumo exclusivo de la cabeza, 
siendo descartado el resto del animal (Taylor, 2004). Esta hipótesis ad-hoc podría explicar, en cierto modo, la elevada frecuencia de ejemplares adultos de Ctenomys en las muestras de TA1, pero se debilita en una evaluación regional, ya que no se verifica en los análisis de egagrópilas actuales del área patagónica. En efecto, muestras numerosas de Patagonia continental con consumos elevados de Ctenomys están dominadas por individuos juveniles o subadultos. Pero no debe desestimarse el factor insular en la conducta trófica de lechuzas. Si bien no existe ningún análisis de egagrópilas actuales efectuado a partir de muestras obtenidas en Tierra del Fuego, el número bajo de sigmodontinos nativos (que son las presas óptimas para estrigiformes en Patagonia; véase Formoso et al. 2016 y la literatura allí revisada) podría estar propiciando un consumo focalizado en ejemplares adultos de Ctenomys y así dar cuenta del registro de TA1.

Finalmente, se destacan dos aspectos con relación a las muestras de niveles holocénicos de TA1. Por un lado, la presencia de grietas y fracturas sobre dientes sugiere un lapso quizá importante de exposición a la intemperie. Por el otro, ya en el campo bioestratinómico, la elevada frecuencia de marcas de raíces indica pedogénesis y desarrollo vegetal afectando al sedimento portador y los elementos contenidos en éste.

\section{Micromamíferos y paleoambientes en TA1}

Tres son los taxones dominantes a lo largo de toda la secuencia de TA1, que en definitiva representa dos segmentos temporales (i.e. Pleistoceno más tardío y Holoceno tardío): Ctenomys magellanicus, Euneomys chinchilloides y Reithrodon auritus. Mientras que la frecuencia del primero presenta una estrecha relación con los tamaños de las muestras, no puede decirse lo mismo para ambos sigmodontinos. E. chinchilloides es el elemento dominante en los niveles pleistocénicos; para el Holoceno tardío, su frecuencia declina gradualmente hasta la actualidad. En cierta forma especular, $R$. auritus está virtualmente ausente durante el Pleistoceno tardío y aumenta, dramáticamente, durante el Holoceno tardío (Fig. 6). Estas diferencias reflejan condiciones ambientales contrastantes.

E. chinchilloides y $R$. auritus conforman un par de especies que puede ser empleado como indicador de la competencia de dos ambientes diferentes. Mientras que el primero es un elemento característico de situaciones de peladal y pedregal muy expuestos a las inclemencias, ambientes decididamente hostiles (e.g. Pearson, 1987; Pardiñas et al. 2011b), el segundo es típico de estepas graminosas (e.g. Pearson, 1988; Pardiñas \& Galliari, 2001). Una base de datos -con más de 500 localidades éditas e inéditas- que recoge gran

Fig. 6. Variación en la abundancia relativa (estimada como NISP\%) de los

cricétidos Euneomys y Reithrodon en las muestras de diferentes capas del sitio arqueológico Tres Arroyos 1 (Tierra del Fuego, Chile).

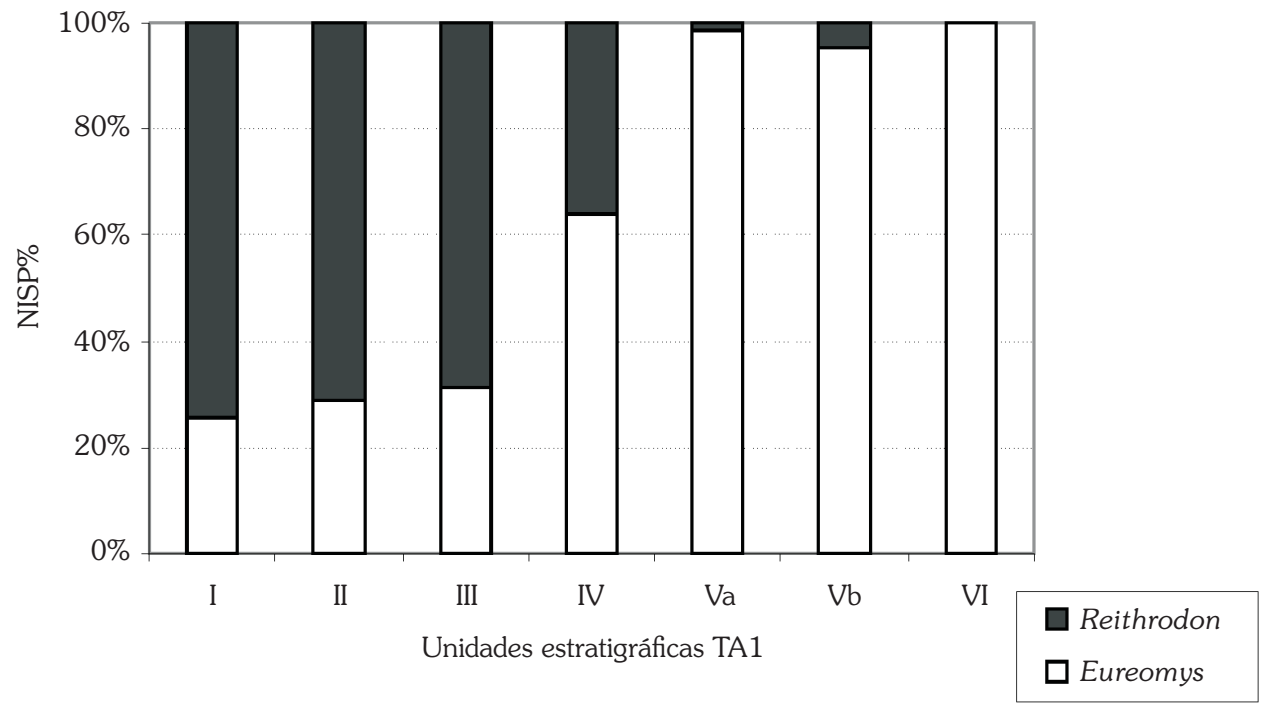


Tabla 8. MNI para Euneomys y Reithrodon y relación Reithrodon/Euneomys en diferentes localidades patagónicas con análisis de egagrópilas actuales de aves rapaces (compilado de varias fuentes). Las localidades se han ordenado en forma creciente según el valor arrojado por dicha relación.

\begin{tabular}{|c|c|c|c|c|c|}
\hline Localidad & Latitud S & Longitud O & Euneomys & Reithrodon & Relación \\
\hline \multicolumn{6}{|l|}{ Alero Destacamento } \\
\hline Guardaparques & $47^{\circ} 50^{\prime}$ & $72^{\circ} 02^{\prime}$ & 63 & 1 & 0,016 \\
\hline \multicolumn{6}{|l|}{ Alero Destacamento } \\
\hline Guardaparques & $47^{\circ} 50^{\prime}$ & $72^{\circ} 02^{\prime}$ & 82 & 2 & 0,024 \\
\hline $3 \mathrm{~km}$ al $\mathrm{N}$ de Cuatro Cerros & $41^{\circ} 28^{\prime}$ & $66^{\circ} 59^{\prime}$ & 46 & 2 & 0,043 \\
\hline Cerro Corona & $41^{\circ} 27^{\prime}$ & $66^{\circ} 54^{\prime}$ & 63 & 3 & 0,048 \\
\hline Estancia San Pedro & $42^{\circ} 04^{\prime}$ & $67^{\circ} 34^{\prime}$ & 36 & 3 & 0,083 \\
\hline \multicolumn{6}{|l|}{ Alero Destacamento } \\
\hline Guardaparques & $47^{\circ} 50^{\prime}$ & $72^{\circ} 02^{\prime}$ & 77 & 10 & 0,130 \\
\hline Subida del Naciente & $41^{\circ} 40^{\prime}$ & $67^{\circ} 09^{\prime}$ & 59 & 8 & 0,136 \\
\hline Sierra de Talagapa & $42^{\circ} 14^{\prime}$ & $68^{\circ} 14^{\prime}$ & 47 & 9 & 0,191 \\
\hline Sierra de Talagapa & $42^{\circ} 12^{\prime}$ & $68^{\circ} 13^{\prime}$ & 129 & 28 & 0,217 \\
\hline Lago Cardiel & $48^{\circ} 50^{\prime}$ & $71^{\circ} 11^{\prime}$ & 50 & 11 & 0,220 \\
\hline Cerro Castillo & $41^{\circ} 58^{\prime}$ & $69^{\circ} 16^{\prime}$ & 28 & 8 & 0,286 \\
\hline Sierra de Talagapa & $42^{\circ} 12^{\prime}$ & $68^{\circ} 13^{\prime}$ & 94 & 32 & 0,340 \\
\hline $4 \mathrm{~km}$ al S de Tres Banderas & $42^{\circ} 48^{\prime}$ & $68^{\circ} 00^{\prime}$ & 131 & 53 & 0,405 \\
\hline Cerro Casa de Piedra 7 & $47^{\circ} 57^{\prime}$ & $72^{\circ} 06^{\prime}$ & 38 & 16 & 0,421 \\
\hline Estancia Monira & $43^{\circ} 42^{\prime}$ & $70^{\circ} 49^{\prime}$ & 46 & 27 & 0,587 \\
\hline Estancia Calcatreo & $41^{\circ} 44^{\prime}$ & $69^{\circ} 22^{\prime}$ & 54 & 64 & 1,185 \\
\hline Río La Leona & $49^{\circ} 54^{\prime}$ & $72^{\circ} 03^{\prime}$ & 37 & 47 & 1,270 \\
\hline Paso del Sapo & $42^{\circ} 41^{\prime}$ & $69^{\circ} 43^{\prime}$ & 48 & 82 & 1,708 \\
\hline Estancia El Gauchito & $45^{\circ} 11^{\prime}$ & $67^{\circ} 11^{\prime}$ & 21 & 42 & 2,000 \\
\hline Estancia Calcatreo & $41^{\circ} 44^{\prime}$ & $69^{\circ} 22^{\prime}$ & 68 & 206 & 3,029 \\
\hline Río Limay & $41^{\circ} 01^{\prime}$ & $71^{\circ} 07^{\prime}$ & 5 & 28 & 5,600 \\
\hline Estancia Pilcañeu & $41^{\circ} 08^{\prime}$ & $70^{\circ} 41^{\prime}$ & 15 & 103 & 6,867 \\
\hline Estancia Tecka & $43^{\circ} 11^{\prime}$ & $71^{\circ} 03^{\prime}$ & 7 & 51 & 7,286 \\
\hline Cerro Leones & $41^{\circ} 04^{\prime}$ & $71^{\circ} 08^{\prime}$ & 23 & 361 & 15,696 \\
\hline Perito Moreno & $41^{\circ} 03^{\prime}$ & $71^{\circ} 00^{\prime}$ & 1 & 31 & 31,000 \\
\hline Cañadón del Tordillo & $40^{\circ} 23^{\prime}$ & $70^{\circ} 11^{\prime}$ & 1 & 32 & 32,000 \\
\hline Paraje La Querencia & $39^{\circ} 07^{\prime}$ & $70^{\circ} 56^{\prime}$ & 2 & 70 & 35,000 \\
\hline
\end{tabular}

parte de los análisis de egagrópilas actuales de estrigiformes generados para la región patagónica (e.g. Pardiñas, 1999a; Formoso et al. 2016), muestra la ausencia virtual de co-dominancia entre este par de especies (Tabla 8). Euneomys es dominante en regiones puntuales caracterizadas por inviernos muy crudos (generalmente con nieve $>6$ meses al año), predominancia de vientos, amplias superficies de roquedales y suelo desnudo y marcada heterogeneidad climática (e.g. altiplanicie de Somuncura por encima de $1.300 \mathrm{~m}$, sector oeste de Santa Cruz en cercanías del ecotono bosqueestepa). Reithrodon es dominante en estepas de pastos cortos, medianamente húmedas y también en estepas arbustivas húmedas, como aquellas que caracterizan el sector patagónico noroccidental. No conocemos las frecuencias de estos taxones en la mitad norte de Tierra del Fuego ni, menos 
aún, en el ámbito local de TA1. La evolución de la relación $R$. auritus/E. chinchilloides en el segmento holocénico representado en TA1 muestra un aumento del primero en más de 20 puntos entre las capas IV y III. Posteriormente, ambos taxones se estabilizan (capas III a I; Fig. 6). Estas relaciones pueden interpretarse como reflejo de la implantación de estepas graminosas desde, al menos, los últimos 1,3 ka. Para el Pleistoceno más tardío, la virtual ausencia de $R$. auritus y la opuesta dominancia de E. chinchilloides sugiere condiciones ambientales extremadamente hostiles.

Las especies de sigmodontinos que acompañan a estos dos taxones robustecen estas inferencias. La desaparición holocénica de Eligmodontia podría estar vinculada con estas condiciones ambientales, pero también con aspectos paleogeográficos. De hecho, todo el conjunto faunístico pleistocénico de TA1 sugiere conexiones íntimas, al menos biogeográficas, con la porción austral continental de América del Sur. Esta continuidad biótica habría estado favorecida por la parcial o total ausencia del estrecho de Magallanes, como barrera geográfica (e.g. McCulloch et al. 2005a, 2005b; McCulloch \& Morello, 2009). En TA1 se ha registrado, además de megafauna hoy extinta, al galictino Lyncodon patagonicus, al félido Leopardus colocolo, los cánidos Dusicyon avus y Lycalopex culpaeus y un clado extinto del camélido Lama guanicoe, además de un ave corredora Rheidae (e.g. Prieto \& Canto, 1997; Latorre, 1998; Martin et al. 2009; Weinstock et al. 2009; Martin, 2013; Prevosti et al. 2013; Metcalf et al. 2016). Todos estos vertebrados resultan exclusivos del componente pleistocénico. En este contexto, tanto el registro como la posterior extirpación de Eligmodontia, pierden su singularidad. Un desafío diferente resulta inferir las posibles causas detrás de esta extinción general. Prima facie, el establecimiento de la barrera marina, con la consecuente condición insular para el territorio fueguino, podría haber participado en el proceso. Sin embargo, por el momento carecemos de indicadores cronológicos para establecer cuándo se produjo exactamente la desaparición local de Eligmodontia. Incluso, juzgado por continuidad de hábitats, las comunidades de mata negra que orlan los humedales de bahía San Sebastián resultan tan similares a aquellas comunidades de cabo Vírgenes (i.e. el extremo continental sudoriental, donde Eligmodontia es abundante), que plausiblemente podría estar presente este filotino. Los muestreos en territorio insular han sido muy escasos como para descartar en forma taxativa que la ausencia de Eligmodontia no sea un artefacto. Otro representante de esta tribu de roedores, Phyllotis xanthopygus, tiene actualmente su límite austral en las exposiciones rocosas cercanas a monte Aymond (Pardiñas et al. 2009). Pese a su carácter rupícola que le impone limitaciones, podría ser un buen candidato para haber extendido su geonemia a territorio insular durante el Pleistoceno tardío.

Para el Holoceno tardío, el registro de A. lanosa y O. longicaudatus, ambos ausentes hoy día en los conjuntos del norte de la isla, sugiere la expansión de ambientes de bosque y matorral. Nuestros muestreos señalan la ocurrencia de $O$. longicaudatus en parches de bosque unos $50 \mathrm{~km}$ al SSE de TA1 (e.g. CNP 5608, 5981, ambos coleccionados unos $20 \mathrm{~km}$ al S de Ea. San Julio, Tierra del Fuego). En el sector chileno de la isla, los parches de Nothofagus cubren extensamente faldeos de la Ea. río Chico apenas $20 \mathrm{~km}$ al S de TA1. Aunque no tenemos datos de trampeos en estos ambientes, no parece ilógico suponer que albergan poblaciones actuales de $O$. longicaudatus y, quizás, A. lanosa. Para este último, los registros más próximos a TA1 son aquellos reportados por Osgood (1943; véase también Feijoo et al. 2010) para el extremo oriental del lago Fagnano (ca. 160 $\mathrm{km}$ al SE de TA1).

El devenir más reciente de todo el conjunto de TA1 es difícil de establecer, ante la ausencia de un adecuado parámetro moderno para el cerro de Los Onas. Actualmente, el ambiente mantiene matorrales importantes (e.g. Roja Villegas, 2004). Un muestreo en los mismos podría revelar la persistencia de A. lanosa y $O$. longicaudatus. Más allá de estos considerandos, la hipótesis de dos panoramas ambientales decididamente contrastantes, identificables con los segmentos del Pleistoceno más tardío y del Holoceno tardío, es totalmente congruente con los conjuntos de roedores registrados. Vale la pena explorar, entonces, su ajuste con otros archivos paleoclimáticos e inferencias generadas previamente para el sector de estudio y regiones colindantes. 
Existe una bibliografía profusa sobre la evolución del sector correspondiente al estrecho de Magallanes desde el Último Máximo Glacial, período en el cual habría recibido englazamiento por dos lóbulos principales (sobre el propio estrecho y en la región de bahía Inútil), desde su cabecera en cordillera Darwin. La evidencia disponible sugiere que se trató de una evolución compleja, incluso con participación de procesos neotectónicos. A la deglaciación, aparentemente casi abrupta, habría seguido el colapso de un extenso paleolago proglacial (formado allende la Segunda Angostura). La formación del pasaje de mar, más o menos como actualmente aparece, sería un episodio recién del Pleistoceno más tardío, o incluso del Holoceno más temprano (e.g. Coronato et al. 1999; Bentley \& McCulloch, 2005; Bentley et al. 2005; McCulloch et al. 2005a, 2005b; McCulloch \& Morello, 2009; Ponce et al. 2011).

La mayor parte de los fechados radiocarbónicos referidos a los fogones de la capa Va- indica su depositación en torno a los 10,5 ka. Tanto los registros polínicos o de evidencias múltiples de Tierra del Fuego al norte del lago Fagnano, como así también aquellos del extremo continental decididamente extraandino (e.g. laguna Azul, laguna Potrok Aike; Recasens et al. 2012; Zolitschka et al. 2019), proveen valiosa información ambiental para este segmento temporal (e.g. Heusser, 1993; Markgraf, 1993; Musotto et al. 2018 y las referencias allí citadas). Todos coinciden en un entorno hostil, con temperaturas y condiciones de humedad más bajas que las actuales, quizás también bajo una menor influencia de los "westerlies" australes. El norte de la isla comenzaría a mostrar una expansión del bosque de Nothofagus a partir del Holoceno temprano-medio (Heusser, 1993; Markgraf, 1993; Burry et al. 2006).

Dos secuencias continuas aparecen en particular relevantes para inferir las condiciones ambientales imperantes en cerro de Los Onas durante el límite Pleistoceno-Holoceno: Onamonte y laguna Azul. El perfil de Onamonte 1, c. 66 km SSO de TA1, indica dominancia de elementos esteparios para el Pleistoceno tardío-Holoceno medio, con escasa participación de polen arbóreo (Heusser, 1993, 2003). Un registro mucho más acotado cronológicamente (con niveles datados ca. $12 \mathrm{ka}$ ), en la costa norte de bahía Inútil, revela igualmente valores altos de gramíneas y ciperáceas (Heusser, 2003, p. 156). El perfil de laguna Azul, un cráter volcánico inundado en la costa norte del estrecho de Magallanes en proximidades de la Primera Angostura, representa un archivo paleoclimático más o menos continuo para los últimos 16 ka (Zolitschka et al. 2019). La evidencia de múltiples indicadores permite inferir condiciones muy frías y algo más húmedas que las actuales hacia el fin del Pleistoceno, con atenuación de los westerlies australes.

Contra esto, el Holoceno tardío parece representar un segmento climáticamente mucho más amigable, aunque signado por una variabilidad marcada de las precipitaciones. En el registro paleoclimático múltiple de laguna del Carmen, c. $50 \mathrm{~km}$ SE de TA1, se infiere predominio de pastizal, con una tendencia hacia el incremento de matorral hacia el Holoceno más tardío (Borromei et al. 2018). Este sustancial "mejoramiento" del Holoceno terminal respecto del Pleistoceno, se ve reflejado en las comunidades de roedores de TA1, con un palpable incremento de diversidad. Una perceptible variación en la condición del bosque de Nothofagus, desde cerrado a ecotonal (con la estepa), ha sido inferida en base al perfil polínico de la Ea. río Claro, ubicada unos $25 \mathrm{~km}$ al $\mathrm{N}$ del lago Fagnano (Burry et al. 2007). Estos cambios, de menor escala pero igualmente con impacto sobre el medio, podrían ser los que dispararon el incremento de pastizales y la consecuente respuesta de $R$. auritus que se detecta entre capas IV y III de TA1.

Los registros de pequeños mamíferos asociados a dataciones en Tierra del Fuego son escasos. Quizás el de mejor resolución, tanto por su contenido como por el contexto, sea el recientemente estudiado Las Vueltas 1, yacimiento emplazado unos $55 \mathrm{~km}$ al SE de TA1 (Santiago et al. 2016). Allí, los roedores son abundantes en las tres ocupaciones detectadas, pero en la denominada "3ra ocupación", la más superficial y con cronologías en torno a los 0,5 ka, el conjunto de especies permite hacer algunas consideraciones ambientales. Entre los cricétidos, domina $R$. auritus sobre E. chinchilloides (la relación es 5,9; véase Santiago et al. 2016: Tabla 1) y están representados los abrotriquinos $A$. hirta y $A$. 
olivacea. En este conjunto, Ctenomys alcanza valores superlativos, posiblemente sesgado por el consumo antrópico (Santiago et al. 2016). Los roedores de la ocupación superior de Las Vueltas 1 sugieren el desarrollo de estepas graminosas (más que arbustivas), bajo condiciones climáticas similares a las actuales. También indican que el proceso de declinación regional de E. chinchilloides era más marcado en la porción oriental, que en el sector de TA1.

Los micromamíferos de TA1 aparecen como una herramienta poderosa para hacer reconstrucciones ambientales e inferir condiciones paleogeográficas en torno a la evolución cuaternaria del norte de Tierra del Fuego. Sin embargo, también revelan limitaciones importantes. Entre éstas, siempre emerge un claro mensaje: es necesario sumar más evidencias.

\section{AGRADECIMIENTOS}

Esta contribución resulta un producto tardío de un análisis efectuado hace más de dos décadas. D. Podestá participó, oficiosamente, en la separación taxonómica de algunas muestras de TA1. Aspectos relativos a la taxonomía e implicaciones paleoambientales de los conjuntos estudiados fueron discutidos, en su oportunidad, con D. Udrizar y P. Teta, a la sazón doctorandos en el grupo de trabajo del autor senior. Parte de los resultados fueron avanzados en una conferencia brindada en Chile (2007) y se vieron beneficiados con la discusión de A. Barnosky. Los trabajos de excavación arqueológica del sitio Tres Arroyos 1 fueron financiados por la Municipalidad de Porvenir (1981), Instituto de la Patagonia (1983), Universidad de Magallanes (1986), Proyecto FONDECYT 1960027 (1996-1999), dirigidos por M. Massone. Los trabajos de campo ejecutados en Tierra del Fuego y que brindaron datos valiosos para entender el conjunto insular de roedores, contaron con el auspicio de National Geographic Society (Subsidio 7813-05) y CONICET (PIP 6179) y la participación crucial de D. Udrizar, G. D’Elía, J. Pardiñas, E. Lessa y A. Parada. G. Massaferro fue artífice de los mapas que ilustran este trabajo. En una etapa más reciente, recursos económicos parciales fueron derivados de los PICT (Agencia) 2008-547 y 2014-1039 (UFJP) y Consejo Superior de Investigaciones Científicas de Cooperación Internacional, Grant i-COOPB-20287 (FJF). Nuestro agradecimiento especial a la memoria de los señores Carlos Descourvieres, René Lillo, José Soto, Iris Sánchez; a los señores Marcos Martic, José Calbuante, Blanca Curguán y Pedro Mansilla, de Estancia Tres Arroyos y Estancia Serena por su hospitalidad y por facilitar los trabajos de campo. A todas las instituciones y personas mencionadas, el reconocimiento de los autores.

\section{BIBLIOGRAFÍA}

Alberdi, M. T., \& Prieto, A. (2000). Hippidion (Mammalia, Perissodactyla) de las cuevas de las provincias de Magallanes y Tierra del Fuego. Anales del Instituto de la Patagonia, Serie Ciencias Humanas, 28, 147-171.

Allen, J. A. (1901). New South American Muridae and a new Metachirus. Bulletin of the American Museum of Natural History, 14, 405-412.

Andrade, A., \& Fernández, P. M. (2017). Rodent consumption by hunter gatherers in north Patagonian Andean forests (Argentina): Insights from the small vertebrate taphonomic analysis of two late Holocene archaeological sites. Journal of Archaeological Science Reports, 11, 390-399.

Andrews, P. (1990). Owls, Caves and Fossils. Predation, Preservation, and Accumulation of small mammal bones in caves, with an analysis of the Pleistocene Cave faunas from Westbury-sub-Mendip, Somerset, UK. University of Chicago Press, Chicago.

Andrews, P. (1992). The basis for taphonomic research on vertebrate fossils. En Fernández López S. (Ed.), Conferencias de la Reunión de tafonomía y fosilización (pp. 33-43). Madrid: Editorial Complutense.

Avery, D. M. (1982). Micromammals as palaeoenvironmental indicators and an interpretation of the Late Quaternary in the southern Cape province, South Africa. Annals of the South African Museum, 85, 183-374.

Bennett, E.T. (1832). Characters of a new species of otter (Lutra, Erxl.) and of a new species of mouse (Mus, L.) collected in Chili by Mr. Cuming. Proc. Comm. Sci. Correspond. Proceedings of the Zoological Society of London, part II, 1- 4.

Bennett, E.T. (1836). On a second species of Lagotis (Lag. pallipes). Proceedings of the Zoological Society of London, part III, 67-68.

Bentley, M. J., \& McCulloch, R. D. (2005). Impact of neotectonics on the record of glacier and sea level fluctuations, Strait 
of Magellan, southern Chile. Geografiska Annaler Series A Physical Geography, 87, 393-402.

Bentley, M. J., Sugden, D. E., Hulton, N.R., \& McCulloch, R. D. (2005). The landforms and pattern of deglaciation in the Strait of Magellan and Bahía Inútil, southernmost South America. Geografiska Annaler Series A Physical Geography, 87, 313-333.

Bidau, C. J. (2015). Family Ctenomyidae Lesson, 1842: by Genus Ctenomys Blainville, 1826. En J. L. Patton, U. F. J. Pardiñas y G. D'Elía (Eds.), Mammals of South America, Volume 2 Rodents (pp. 818-877). Chicago and London: The University of Chicago Press.

Borrero, L. A. (1979). Excavaciones en el alero "Cabeza de León” Isla Grande de Tierra del Fuego. Relaciones de la Sociedad Argentina de Antropología, 13, 255271.

Borrero, L. A. (2003). Taphonomy of the Tres Arroyos 1 Rockshelter, Tierra del Fuego, Chile. Quaternary International, 109-110, 87-93.

Borrero, L. A., \& Casiraghi, M. (1980). Excavaciones en el sitio Bloque Errático I (San Sebastián, Tierra del Fuego). Relaciones de la Sociedad Argentina de Antropología, 14, 129-142.

Borromei, A. M., Candel, M. S., Musotto, L. L., Cusminsky, G., Martínez, M. A., Coviaga, C. A., Ponce, J. F., \& Coronato, A. (2018). Late Holocene wet/dry intervals from Fuegian steppe at Laguna Carmen, southern Argentina, based on a multiproxy record. Palaeogeography, Palaeoclimatology, Palaeoecology, 499, 56-71.

Braun, J. K., \& Pardiñas, U. F. J. (2015). Genus Euneomys Coues, 1874. En J. L. Patton, U. F. J. Pardiñas y G. D'Elía (Eds.), Mammals of South America, Volume 2 Rodents (pp. 83-89). Chicago and London: The University of Chicago Press.

Burry, L. S., Trivi de Mandri, M. E., \& D’Antoni, H. L. (2006). Paleocomunidades vegetales del centro de Tierra del Fuego durante el Holoceno temprano y tardío. Revista Museo Argentino Ciencias Naturales, 8, 127-133.

Burry, L. S., Trivi de Mandri, M. E., \& D'Antoni, H. L. (2007).

Modern analogues and past environments in central Tierra del Fuego, Argentina. Anales del Instituto de la Patagonia, 35, 5-14.

Caviglia, S. E. (1985-1986). Nuevos restos de cánidos tempranos en sitios arqueológicos de Fuego-Patagonia. Anales del Instituto de la Patagonia, Serie Ciencias Sociales, 16, 85-93.

Chebez, J.C., Pardiñas, U. F. J., \& Teta, P. (2014). Mamíferos terrestres de la Patagonia, sur de Argentina y Chile.
Editorial Vázquez Mazzini, Buenos Aires.

Coronato, A., Salemme, M., \& Rabassa, J. (1999). Palaeoenvironmental conditions during the early peoplin of southernmost South America (Late Glacial-Early Holocene, 14-8 ka B.P.). Quaternary International, 53/54, 77-92.

Fasanella, M., Bruno, C., Cardoso, Y. T., \& Lizarralde, M. (2013). Historical demography and spatial genetic structure of the subterranean rodent Ctenomys magellanicus in Tierra del Fuego (Argentina). Zoological Journal of the Linnean Society, 169, 697-710.

Feijoo, M., D’Elía, G., Pardiñas, U. F. J., \& Lessa, E. P. (2010). Systematics of the southern Patagonian-Fueguian endemic Abrothrix lanosus (Rodentia: Sigmodontinae): phylogenetic position, karyotypic and morphological data. Mammalian Biology, 75, 122-137.

Fernández, F.J. (2012). Microvertebrados del Holoceno de sitios arqueológicos en el sur de Mendoza (República Argentina): aspectos tafonómicos y sus implicancias en la subsistencia humana. Tesis de doctorado. Facultad de Ciencias Naturales y Museo, SEDICI, Repositorio online de la Universidad Nacional de La Plata: http://sedici.unlp.edu.ar/handle/10915/21965

Fernández, F. J., Teta, P., Barberena, R., \& Pardiñas, U. F. J. (2012). Small Mammal Remains from Cueva Huenul 1, Argentina. Taphonomy and Palaeoenvironments since the Late Pleistocene. Quaternary International, 278, 22-31.

Fernández, F. J., Teta. P., \& Pardiñas, U. F. J. (2017a). Evidencias arqueológicas de explotación antrópica de micromamíferos en el extremo austral de América del Sur. Anales de Arqueología y Etnología, 72, 9-32.

Fernández, F. J., Montalvo, C. I., Fernández-Jalvo, Y., Andrews, P., \& López, J. M. (2017b). A re-evaluation of the taphonomic methodology for the study of small mammal fossil assemblages of South America. Quaternary Science Reviews, 155, 37-49.

Fernández, F. J., Hadler, P., Cherem, J., Saldanha, J., Stutz, N., Dias, A., \& Pardiñas, U. F. J. (2019). Holocene small mammals hunted by owls and humans in southern Brazil: taphonomic evidence and biological significance. Boreas. DOI 10.1111/bor. 12399

Fernández-Jalvo, Y., \& Andrews, P. (1992). Small mammal taphonomy of Gran Dolina, Atapuerca (Burgos), Spain. Journal of Archaeological Science, 19, 407-428.

Fischer, G. (1814). Zoognosia tabulis synopticis illustrata. Volumen tertium. Quadrupedum reliquorum, cetorum et montrymatum descriptionem continens. Nicolai Sergeidis Vsevolozsky, Moscow. 
Formoso, A., Teta, P., Carbajo, A., \& Pardiñas, U. F. J. (2016). Unraveling the patterns of small mammal species richness in the southernmost aridlands of South America. Journal of Arid Environments, 134, 136144.

Galliari, C. A., \& Pardiñas, U. F. J. (1999). Abrothrix lanosus (Rodentia, Muridae) en la Patagonia continental, Argentina. Neotropica, 45, 119-120.

Guzmán Sandoval, J. (2010). Mammalia, Rodentia, Sigmodontinae, Abrothrix lanosus (Thomas, 1897): Topotype, distribution, and new locality records for Chile. Check List, 6, 383-386.

Hadly, E. A. (1996). Influence of Late-Holocene climate on Northern Rocky Mountain mammals. Quaternary Research, 46, 298-310.

Hajduk, A., Albornoz, A. M., \& Lezcano, M. (2004). El "Mylodon" en el patio de atrás. Informe preliminar sobre los trabajos en el sitio El Trébol, ejido urbano de San Carlos de Bariloche, Pcia. de Río Negro. En Actas de las V Jornadas de Arqueología de la Patagonia, Contra Viento y Marea, Arqueología de Patagonia (pp. 715-731). Ciudad Autónoma de Buenos Aires: Instituto Nacional de Antropología y Pensamiento Latinoamericano, Sociedad Argentina de Antropología.

Heusser, C. (1993). Late Quaternary forest-steppe contact zone, Isla Grande de Tierra del Fuego, Subantarctic South America. Quaternary Science Reviews, 12, 169-177.

Heusser, C. (2003). Ice Age southern Andes: A chronicle of palaeoecological events. Elsevier Science, Amsterdam.

Laming-Emperaire, A. (1972). Sites préhistoriques de Patagonie chilienne. Objets et Mondes, XII (2), 201-224.

Lanzone, C., Braun, J. K., Patton, J. L., \& Pardiñas, U. F. J. (2015). Genus Eligmodontia F. Cuvier, 1837. En J. L. Patton, U. F. J. Pardiñas y G. D'Elía (Eds.), Mammals of South America, Volume 2 Rodents (pp. 508-522). Chicago and London: The University of Chicago Press.

Latorre, C. (1998). Paleontología de mamíferos del alero Tres Arroyos 1, Tierra del Fuego, XII Región, Chile. Anales del Instituto de la Patagonia, Serie Ciencias Naturales, 26, 77-90.

Lessa, E. P., D’Elía, G., \& Pardiñas, U. F. J. (2010). Genetic footprints of late Quaternary climate change in the diversity of Patagonian-Fueguian rodents. Molecular Ecology, 19, 3031-3037.

Linnaeus, C. (1758). Systema naturae per regnum tria naturae, secundum classes, ordines, genera, species, cum characteribus, differentiis, synonymis, locis. Laurentii Salvii, 1, Editio decima, reformata, Holmiae.

Lyman, R. L. (1994). Relative abundances of skeletal specimens and taphonomic analysis of vertebrate remains. Palaios, 9, 288-298.

Mann Fisher, G. (1978). Los pequeños mamíferos de Chile. Marsupiales, quirópteros, edentados y roedores. Gayana Zoología, 40, 1-342.

Marconi, P. N. (1988). Efecto de las perturbaciones intensas sobre la estructura de las comunidades de roedores. Tesis de doctorado. Facultad de Ciencias Exactas y Naturales, Universidad Nacional de Buenos Aires.

Mares, M. A., Braun, J. K., Coyner, B. S., \& van den Bussche, R. A. (2008). Phylogenetic and biogeographic relationships of gerbil mice Eligmodontia (Rodentia, Cricetidae) in South America, with a description of a new species. Zootaxa, 1753, 1-33.

Markgraf, V. (1993). Palaeoenvironments and palaeoclimates in Tierra del Fuego and southernmost Patagonia, South America. Palaeogeography, Palaeoclimatology, Palaeoecology, 102, 53-68.

Martın, F. (1997). Cerro de los Onas: Análisis de roedores de Tres Arroyos 1 (TA1). Hombre temprano y paleoambiente en Tierra del Fuego. Informe de Avance Proyecto FONDECYT 1960027. Manuscrito inédito.

Martin, F. (2013). Tafonomía y paleoecología de la Transición Pleistoceno-Holoceno en FuegoPatagonia. Interacción entre humanos y carnívoros y su importancia como agentes en la formación del registro fósil. Ediciones de la Universidad de Magallanes, Punta Arenas.

Martin, F. M., Massone, M., Prieto, A., \& Cárdenas, P. (2009). Presencia de Rheidae en Tierra del Fuego durante la Transición Pleistoceno-Holoceno. Implicancias biogeográficas y paleoecológicas. Magallania, 37(1), 173-177.

Massoia, E. (1983). La alimentación de algunas aves del orden Strigiformes. Hornero, 12, Nro. Extraordinario, 119124.

Massoia, E., \& Chebez, J. C. (1993). Mamíferos silvestres del Archipiélago Fuegino. L.O.L.A. (Literature of Latin America), Santa Fe.

Massone, M. (1987). Los cazadores paleoindios de Tres Arroyos (Tierra del Fuego). Anales del Instituto de la Patagonia, Serie Ciencias Sociales, 17, 47-60.

Massone, M. (1988). Artefactos óseos del yacimiento arqueológico Tres Arroyos (Tierra del Fuego). Anales del Instituto de la Patagonia, Serie Ciencias Sociales, 18, 107-112.

Massone, M. (2004). Los cazadores después del hielo. Colección de Antropología VII. Centro de Investigaciones Diego 
Barros Arana, Dirección de Bibliotecas, Archivos y Museos, Santiago.

Massone, M. (2010). Abrigos rocosos de Magallanes en la transición Pleistoceno-Holoceno. Actas XVII Congreso Nacional de Arqueología Chilena Valdivia 2006. Tomo 2, 729-740, Valdivia.

Massone, M., Prieto, A., Jackson, D., Cárdenas, G., Arroyo, M., \& Cárdenas P. (1998). Los cazadores tempranos y sus fogatas: una nueva historia para la cueva Tres Arroyos 1. Tierra del Fuego. Boletín de la Sociedad Chilena de Arqueología, 26, 11-18.

McCulloch, R., \& Morello, F. (2009). Evidencia glacial y paleoecológica de ambientes tardiglaciales y del Holoceno temprano. Implicaciones para el poblamiento temprano de Tierra del Fuego. En M. Salemme, F. Santiago, M. Álvarez, E. Piana, M. Vázquez \& M. E. Mansur (Eds.), Arqueología de Patagonia: una mirada desde el último confín (pp. 119-136). Ushuaia: Editorial Utopías.

McCulloch, R. D., Bentley, M. J., Tipping, R. M., \& Clapperton, C. M. (2005a). Evidence for late-glacial ice dammed lakes in the central Strait of Magellan and Bahía Inútil, southernmost South America. Geografiska Annaler Series A Physical Geography, 87, 335-362.

McCulloch, R.D., Fogwill, C.J., Sugden, D.E., Bentley, M.J., \& Kubik, P.W. (2005b). Chronology of the last glaciation in central Strait of Magellan and Bahía Inútil, southernmost South America. Geografiska Annaler Series A Physical Geography, 87, 289-312.

Mengoni Goñalons, G. L. (1987). Modificaciones culturales y animales en los huesos de los niveles inferiores del sitio Tres Arroyos 1 (Tierra del Fuego, Chile). Anales del Instituto de la Patagonia, Serie Ciencias Sociales, 17, 61-66.

Metcalf, J. L., Turney, C., Barnett, R., Martin, F., Bray, S., Vilstrup, J., Orlando, L., Salas-Gismondi, R., Loponte, D., Medina, M., De Nigris, M., Civalero, M., Fernández, P., Gasco, A., Durán, V., Seymour, K., Otaola, C., Gil, A., Paunero, R., Prevosti, F., Bradshaw, C., Wheeler, J., Borrero, L., Austin, J., \& Cooper, A. (2016). Synergistic roles of climate warming and human occupation in Patagonian megafaunal extinctions during the Last Deglaciation. Science Advances, 2, 1-8.

Montalvo, C. I., \& Fernández, F. J. (2019). Review of the actualistic taphonomy of small mammals ingested by South American predators. Its importance in the interpretation of the fossil record. Publicación Electrónica de la Asociación Paleontológica
Argentina, 19, 18-46.

Moore, D. M. (1983). Flora of Tierra del Fuego. A. Nelson, England, Missouri Botanical Garden USA.

Muñoz, A. S. (2000). El procesamiento de guanacos en Tres Arroyos 1, Isla Grande de Tierra del Fuego. En Desde el país de los gigantes. Perspectivas arqueológicas en Patagonia (pp. 499-517). Río Gallegos: Universidad de la Patagonia Austral.

Musotto, L. L., Candel, M. S., Borromei, A. M., Ponce, F. J., \& Bianchinotti, M. V. (2018). La palinología como una herramienta para la caracterización de paleoambientes continentales y marinos del Cuaternario tardío en el Archipiélago de Tierra del Fuego. Publicación Electrónica de la Asociación Paleontológica Argentina, 18, 131-155.

Olrog, C. (1950). Mamíferos y aves del archipiélago de Cabo de Hornos. Acta Zoológica Lilloana, 9, 505-532.

Ojeda, A., Novillo, A., Ovejero, R. J., Cuello, P. A., Lanzone, C., Borisenko, A. V., Braun, J. K., Mares, M. A., \& Ojeda, R. (2015). A cytogenetic, molecular genetic and morphological study of Patagonian chinchilla mice Euneomys (Rodentia, Cricetidae) in the Southern Central Andes. Mammal Research, 60, 61-69.

Osgood, W. H. (1943). The mammals of Chile. Field Museum of Natural History, Zoological series, 30, 1-268.

Palma, R. E., \& Rodríguez-Serrano, E. (2018). Systematics of Oligoryzomys (Rodentia, Cricetidae, Sigmodontinae) from southern Chilean Patagonia, with the description of a new species. Journal of Zoological Systematics and Evolutionary Research, 56, 280-299.

Pardiñas, U. F. J. (1999a). Los roedores muroideos del Pleistoceno Tardío-Holoceno en la Región Pampeana (sector este) y Patagonia (República Argentina): aspectos taxonómicos, importancia bioestratigráfica y significación paleoambiental. Tesis de doctorado. Facultad de Ciencias Naturales y Museo, Universidad Nacional de La Plata.

Pardiñas, U. F. J. (1999b). Tafonomía de microvertebrados en yacimientos arqueológicos de Patagonia (Argentina). Arqueología, 9, 265-340.

Pardiñas, U. F. J., \& Galliari, C. A. (2001). Reithrodon auritus. Mammalian Species, 664, 1-8.

Pardiñas, U. F. J., \& Teta, P. (2008). Small mammals and palaeoenvironments around the Pleistocene-Holocene boundary in Patagonia. Current Research in the Pleistocene, 25, 30-32.

Pardiñas, U. F. J., \& Teta, P. (2013). Holocene stability and recent dramatic changes in micromammalian communities of northwestern Patagonia. Quaternary 
International, 305, 127-140.

Pardiñas, U. F. J., Teta, P., Cirignoli, S., \& Podestá, D. H. (2003). Micromamíferos (Didelphimorphia y Rodentia) de Norpatagonia Extra Andina, Argentina: taxonomía alfa y biogeografía. Mastozoología Neotropical, 10, 69-113.

Pardiñas, U. F. J., Udrizar Sauthier, D., \& Teta, P. (2009). Roedores del extremo sudoriental continental de Argentina. Mastozoología Neotropical, 16, 471-473.

Pardiñas, U. F. J., Teta, P., Formoso, A., \& Barberena, R. (2011a). Roedores del extremo austral: tafonomía, diversidad y evolución ambiental durante el Holoceno tardío. En L. A. Borrero y K. Borrazzo (Eds.), Bosques, montañas y cazadores. Investigaciones Arqueológicas en Patagonia Meridional (pp. 61-84). Ciudad Autónoma de Buenos Aires: Editorial Dunken.

Pardiñas, U. F. J., Teta, P., D'Elía, G., \& Lessa, E. (2011b). The evolutionary history of sigmodontine rodents in Patagonia and Tierra del Fuego. Biological Journal of the Linnean Society, 103, 495-513.

Pardiñas, U. F. J., Galliari, C. A., \& Teta, P. (2015). Tribe Reithrodontini Vorontsov, 1959. En J. L. Patton, U. F. J. Pardiñas y G. D'Elía (Eds.), Mammals of South America, Volume 2 Rodents (pp. 559-565). Chicago and London: The University of Chicago Press.

Patton, J. L., Pardiñas, U. F. J., \& D’ elía, G. (Eds.) (2015). Mammals of South America, vol. 2 Rodents. London and Chicago: The University of Chicago Press.

Patterson, B. D., Gallardo, M. H., \& Freas, K. E. (1984). Systematics of mice of the subgenus Akodon (Rodentia: Cricetidae) in southern South America, with the description of a new species. Fieldiana Zoology, 23, $1-16$.

Pearson, O. (1987). Mice and the Postglacial history of the Traful valley of Argentina. Journal of Mammalogy, 68, 469478.

Pearson, O. (1988). Biology and feeding dynamics of a South American herbivorous rodent, Reithrodon. Studies on Neotropical Fauna \& Environment, 23, 25-39.

Pearson, O. (1995). Annotated keys for identifying small mammals living in or near Nahuel Huapi National Park or Lanin National Park, southern Argentina. Mastozoología Neotropical, 2, 99-148.

Pearson, O., \& Christie, M. (1991). Sympatric species of Euneomys (Rodentia, Cricetidae). Studies on Neotropical Fauna and Environment, 26, 121-127.

Pearson, O., \& Pearson, A. K. (1993). La fauna de mamíferos pequeños cerca de Cueva Traful I, Argentina: pasado y presente. Præhistoria, 1, 73-89.
Pearson, O., \& Smith, M. F. (1999). Genetic similarity between Akodon olivaceus and Akodon xanthorhinus (Rodentia: Muridae) in Argentina. Journal of Zoology, 247, 43-52

Pine, R., Miller, S., \& Schamberger, M. (1979). Contributions to the mammalogy of Chile. Mammalia, 43, 339-376.

Ponce, J., Borromei, A., Rabassa, J., \& Martínez, O. (2011). Late Quaternary palaeoenvironmental change in western Staaten Island $\left(54.5^{\circ} \mathrm{S}, 64^{\circ} \mathrm{W}\right)$, Fuegian Archipelago. Quaternary International, 233, 89-100.

Prevosti, F., Martin, F., \& Massone, M. (2013). First record of Smilodon lund (Felidae, Machairodontinae) in Tierra del Fuego island (Chile). Ameghiniana, 50, 605-610.

Prieto, A., \& Canto, J. (1997). Presencia de un lamoide atípico en la Cueva Lago Sofía 4 (Última Esperanza) y Tres Arroyos (Tierra del Fuego) región de Magallanes, Chile. Anales del Instituto de la Patagonia, Serie Ciencias Humanas, 25, 147-150.

Rau, J., Yáñez, J., \& Jaksic, F. (1978). Confirmación de Notiomys macronyx alleni O. y Eligmodontia typus typus C., y primer registro de Akodon (Abrothrix) lanosus (Rodentia: Cricetidae) en la zona de Última Esperanza (XII Región, Magallanes). Anales del Instituto de la Patagonia (Chile), 9, 203-204.

Recasens, C., Ariztegui, D., Gebhardt, C., Gogorza, C., Haberzettl, T., Hahn, A., Kliem, P., Lisé-Pronovost, A., Lücke, A., Maidana, N., Mayr, C., Ohlendorf, C., Schäbitz, F., St-Onge, G., Wille, M., Zolitschka, B., \& The PASADO Science Team (2012). New insights into palaeoenvironmental changes in Laguna Potrok Aike, southern Patagonia, since the Late Pleistocene: The PASADO multiproxy record. Holocene, 22, 13231335.

Raise, D., \& Gallardo, M. H. (1990). A taxonomic study of the South American genus Eunemys (Cricetidae, Rodentia). Revista Chilena de Historia Natural, 63, 73-82.

Reise, D., \& Venegas, W. (1987). Catalogue of records, localities, and biotopes from research work on small mammals in Chile and Argentina. Gayana, Zoología, 51, 103-130.

Reyes, O., Méndez, C., Trejo, V., \& Velásquez, H. (2007). El Chueco 1: un asentamiento multicomponente en la estepa occidental de Patagonia central (11.400 a 2.700 años cal. AP, $\sim 44^{\circ}$ S). Magallania, 35, 107-119.

Roja Villegas, G. (2004). Estudios botánicos, paleoambiente y arqueología. Cerro Onas, Tres Arroyos, Tierra del Fuego. Chungara, volumen especial, 381-386.

Saavedra, B., \& Simonetti, J. A. (1998). Small mammal 
taphonomy: intraspecific bone assemblage comparison between South and North American barn owl, Tyto alba, populations. Journal of Archaeological Science, 25, 165-170.

Santiago, F. C., Salemme, M., \& Pardiñas, U. F. J. (2016). Análisis de restos de roedores del sitio arqueológico Las Vueltas 1, Tierra del Fuego, Argentina. Arqueología, 22, 211-230.

Saxon, E. (1979). Natural Prehistory: The Archaeology of Fuego-Patagonian Ecology. Quaternaria, 21, 329356.

Shotwell, J. A. (1955). An approach to the paleoecology of mammals. Ecology, 36, 327-337.

Simonetti, J. A. (1989). Small mammals as palaeoenvironmental indicators: validation for species of central Chile. Revista Chilena de Historia Natural, 62, 109-114.

Simonetti, J. A., \& Rau, J. R. (1989). Roedores del Holoceno Temprano de la Cueva del Milodón, Magallanes, Chile. Noticiario Mensual del Museo Nacional de Historia Natural de Chile, 315, 3-5.

Tammone, M., Hajduk, A., Arias, P., Teta, P., Lacey, E., \& Pardiñas, U.F.J. (2014). Last Glacial Maximum environments in northwestern Patagonia revealed by fossil small mammals. Quaternary Research, 82, 198208.

Taylor, I. (2004). Barn Owls: Predator-Prey relationships and conservation. Cambridge University Press, Cambridge.

Teta, P., \& Pardiñas, U. F. J. (2014). Variación morfológica cualitativa y cuantitativa en Abrothrix longipilis Cricetidae, Sigmodontinae). Mastozoología Neotropical, 21, 291-309.

Thomas, O. (1895). Descriptions of four small mammals from South America, including one belonging to the peculiar marsupial genus "Hyracodon". Tomes. The Annals and Magazine of Natural History, series 10, 16, 367-370.

Thomas, O. (1897). On some small mammals from Salta, northern Argentina. The Annals and Magazine of Natural History, series 6, 20, 214-218.

Thomas, O. (1908). A new Akodon from Tierra del Fuego. The Annals and Magazine of Natural History, series 8, 2, 496-497.

Waterhouse, G. R. (1837). Numerous species of Mus, forming part of the collection presented by Charles Darwin, from the southern coast of South America. Proceedings of the Zoological Society of London, 1837, 15-21.

Waterhouse, G. R. (1839). Mammalia. En C. Darwin (Ed.), The zoology of the Voyage of the H.M.S. Beagle under the command of Captain FitzRoy, R.N., during the Years 1832-1836 (pp. 49-97). London: Smith, Elder and Co.

Weinstock, J., Shapiro, B., Prieto, A., Marín, J. C., González, B. A., Gilbert, T., \& Willerslev, E. (2009). The Late Pleistocene distribution of vicuñas (Vicugna vicugna) and the "extinction" of the gracile llama ("Lama gracilis"): New molecular data. Quaternary Science Reviews, 28, 1369-1373.

Weksler, M., \& Bonvicino, C. R. (2015). Genus Oligoryzomys Bangs, (1900). En J. L. Patton, U. F. J. Pardiñas y G. D'Elía (Eds.), Mammals of South America, Volume 2 Rodents (pp. 417-437). Chicago and London: The University of Chicago Press.

Zolitschka, B., Fey, M., Janssen, S., Maidana, N., Mayr, C., Wulf, S., Haberzettl, T., Corbella, H., Lücke, A., Ohlendorf, C., \& Schäbitz, F. (2019). Southern Hemispheric Westerlies control sedimentary processes of Laguna Azul (south-eastern Patagonia, Argentina). The Holocene, 29, 403-420. 
Anexo 1. NISP detallado por muestra (cuadrícula A/B) para la secuencia de micromamíferos estudiada del sitio Tres Arroyos 1 (Tierra del Fuego, Chile). La información estratigráfica fue extractada de las etiquetas originales del material (profundidades en centímetros; "corurera"=madriguera asignada tentativamente a Ctenomys [coruro]).

Entre corchetes denota resto considerado intrusivo. Abreviaturas: $\mathrm{C}=$ cráneo, $\mathrm{P}=$ paladar, $\mathrm{HMD}=$ hemimaxilar derecho, $\mathrm{HMI}=$ hemimaxilar izquierdo, $\mathrm{RMD}=$ hemimandíbula derecha, $\mathrm{RMI}=$ hemimandíbula izquierda.

\begin{tabular}{|c|c|c|c|c|c|c|c|c|}
\hline Capa & Profundidad & Taxón & $\mathrm{C}$ & $\mathrm{P}$ & HMD & HMI & RMD & RMI \\
\hline I & $0-5$ & C. magellanicus & 2 & 1 & 1 & 1 & 5 & 5 \\
\hline I & $0-5$ & E. chinchilloides & & & 1 & & 5 & 6 \\
\hline I & $0-5$ & R. auritus & & & 7 & 9 & 10 & 9 \\
\hline II & $-5-10$ & C. magellanicus & 4 & 1 & 1 & & 7 & 8 \\
\hline II & $-5-10$ & E. chinchilloides & 1 & & & 1 & 9 & 2 \\
\hline II & $-5-10$ & O. longicaudatus & & & & & & 1 \\
\hline II & $-5-10$ & O. cuniculus & & & & & [2] & [1] \\
\hline II & $-5-10$ & R. auritus & & & 9 & 12 & 13 & 14 \\
\hline III & $-24-29$ & A. lanosa & & & & & 3 & 3 \\
\hline III & $-24-29$ & A. hirta & & & & & 4 & \\
\hline III & $-24-29$ & A. olivacea & 1 & & 1 & & 4 & 5 \\
\hline III & $-24-29$ & C. magellanicus & 26 & 10 & 4 & 2 & 44 & 35 \\
\hline III & $-24-29$ & E. chinchilloides & 4 & 2 & 16 & 32 & 70 & 47 \\
\hline III & $-24-29$ & O. longicaudatus & & & & & 1 & 1 \\
\hline III & $-24-29$ & O. cuniculus & & & & [1] & [1] & [1] \\
\hline III & $-24-29$ & R. auritus & 1 & & 86 & 95 & 101 & 81 \\
\hline IVa & Prof. real -34-49 & C. magellanicus & 5 & 1 & & & 12 & 6 \\
\hline $\mathrm{IVa}$ & Prof. real -34-49 & E. chinchilloides & & & & & 7 & 5 \\
\hline $\mathrm{IVa}$ & Prof. real -34-49 & R. auritus & & & 5 & 3 & 3 & 6 \\
\hline IV & - & A. lanosa & & & & & 1 & 1 \\
\hline IV & - & A. hirta & & & & & & 1 \\
\hline IV & - & A. olivacea & & & & 1 & 5 & 8 \\
\hline IV & - & C. magellanicus & 10 & 9 & 1 & 1 & 34 & 32 \\
\hline IV & - & E. chinchilloides & 2 & 3 & 100 & 86 & 194 & 199 \\
\hline IV & - & O. longicaudatus & & & 1 & & & \\
\hline IV & - & O. cuniculus & & & & & 1 & \\
\hline IV & - & R. auritus & 2 & & 40 & 48 & 44 & 44 \\
\hline $\mathrm{V}$ & limpieza "corurera" & A. lanosa & & & & 1 & & \\
\hline $\mathrm{V}$ & limpieza "corurera" & A. olivacea & & & & & 5 & 5 \\
\hline $\mathrm{V}$ & limpieza "corurera" & C. magellanicus & 2 & 4 & & & 9 & 14 \\
\hline V & limpieza "corurera" & E. chinchilloides & & & 47 & 53 & 82 & 72 \\
\hline $\mathrm{V}$ & limpieza "corurera" & O. cuniculus & 1 & & & & & 1 \\
\hline
\end{tabular}




\begin{tabular}{|c|c|c|c|c|c|c|c|c|}
\hline Capa & Profundidad & Taxón & C & $\mathrm{P}$ & HMD & HMI & RMD & RMI \\
\hline V & limpieza "corurera" & R. auritus & & & 8 & 8 & 8 & 10 \\
\hline Va & - & A. olivacea & & & 1 & & 2 & \\
\hline $\mathrm{Va}$ & - & C. magellanicus & 1 & 4 & 2 & 1 & 4 & 7 \\
\hline $\mathrm{Va}$ & - & E. chinchilloides & 3 & & 27 & 18 & 47 & 44 \\
\hline Va & - & R. auritus & & & [1] & $2[1]$ & & 3 \\
\hline Va inferior & - & A. hirta & & & & & & 1 \\
\hline Va inferior & - & C. magellanicus & & & & & 2 & 1 \\
\hline Va inferior & - & E. chinchilloides & & & 2 & 2 & 6 & 8 \\
\hline Va inferior & - & R. auritus & & & & [1] & {$[1]$} & \\
\hline Vb superior & - & E. chinchilloides & & & 2 & & 5 & 1 \\
\hline Vb inferior & - & E. chinchilloides & & & & & 2 & 2 \\
\hline $\mathrm{Vb}$ inferior & - & C. magellanicus & & & 1 & & 2 & \\
\hline
\end{tabular}

Anexo 2. NISP detallado por muestra (cuadrícula D) para la secuencia de micromamíferos estudiada del sitio Tres Arroyos 1 (Tierra del Fuego, Chile). Para las referencias, véase el Anexo 1.

\begin{tabular}{|c|c|c|c|c|c|c|c|c|}
\hline Capa & Profundidad & Taxón & C & $\mathrm{P}$ & HMD & HMI & RMD & RMI \\
\hline II & $-6-8$ a -14-15 & A. lanosa & & & & & 1 & \\
\hline II & $-6-8 a-14-15$ & A. olivacea & & & & & 1 & \\
\hline II & $-6-8 a-14-15$ & C. magellanicus & 21 & 10 & 4 & 5 & 47 & 43 \\
\hline II & $-6-8 a-14-15$ & E. chinchilloides & 2 & 1 & 10 & 9 & 51 & 34 \\
\hline II & $-6-8$ a -14-15 & R. auritus & & & 60 & 53 & 73 & 62 \\
\hline III superior & -15 a $-20-22$ & A. olivacea & & & & & 1 & \\
\hline III superior & -15 a $-20-22$ & C. magellanicus & 21 & 10 & 4 & 4 & 43 & 47 \\
\hline III superior & -15 a $-20-22$ & E. chinchilloides & & 6 & 10 & 15 & 33 & 41 \\
\hline III superior & -15 a $-20-22$ & R. auritus & & & 67 & 62 & 51 & 63 \\
\hline IVa & - & A. olivacea & & & & & & 1 \\
\hline IVa & - & C. magellanicus & 18 & 10 & 5 & 4 & 64 & 42 \\
\hline $\mathrm{IVa}$ & - & E. chinchilloides & 2 & & 11 & 13 & 86 & 87 \\
\hline IVa & - & O. cuniculus & & & [1] & {$[1]$} & & \\
\hline IVa & - & R. auritus & & & 77 & 60 & 50 & 60 \\
\hline $\mathrm{IVb}$ & parte basal -45-55-60 & C. magellanicus & 3 & 4 & 2 & 1 & 12 & 22 \\
\hline $\mathrm{IVb}$ & parte basal -45-55-60 & E. chinchilloides & 1 & & 2 & 3 & 28 & 33 \\
\hline $\mathrm{IVb}$ & parte basal -45-55-60 & O. cuniculus & & & {$[1]$} & & [3] & {$[1]$} \\
\hline $\mathrm{IVb}$ & parte basal -45-55-60 & R. auritus & & & 19 & 9 & 15 & 10 \\
\hline $\mathrm{IVb}$ & -45 a $55-60-70$ & R. magellanicus & & & & & 4 & 8 \\
\hline
\end{tabular}




\begin{tabular}{|c|c|c|c|c|c|c|c|c|}
\hline Capa & Profundidad & Taxón & $\mathrm{C}$ & $\mathrm{P}$ & HMD & HMI & RMD & RMI \\
\hline $\mathrm{IVb}$ & -45 a $55-60-70$ & E. chinchilloides & & & 1 & & 10 & 13 \\
\hline $\mathrm{IVb}$ & -45 a $55-60-70$ & R. auritus & & & {$[1]$} & $2[1]$ & $3[1]$ & $2[1]$ \\
\hline IV-V & - & C. magellanicus & & & & & 1 & \\
\hline $\mathrm{Va}$ & - & A. olivacea & & & & & 1 & 1 \\
\hline Va & - & C. magellanicus & $3[2]$ & 6 & {$[1]$} & $3[1]$ & 45 & 34 \\
\hline Va & - & E. chinchilloides & 2 & & 23 & 29 & 171 & 175 \\
\hline Va & - & O. cuniculus & & & [1] & [1] & {$[1]$} & \\
\hline $\mathrm{Va}$ & - & R. auritus & & & [24] & [24] & [23] & [14] \\
\hline Va superior & - & C. magellanicus & & & & & 3 & 2 \\
\hline Va superior & - & E. chinchilloides & & & 2 & 1 & 5 & 7 \\
\hline Va superior & - & R. auritus & & & & & & 1 \\
\hline Va inferior & - & C. magellanicus & & & & & & 1 \\
\hline Va inferior & - & E. chinchilloides & & & & 1 & 1 & \\
\hline $\begin{array}{c}\text { Va base a sup } \\
\qquad b\end{array}$ & $-75-93$ & C. magellanicus & & & 1 & 1 & 3 & \\
\hline $\begin{array}{c}\text { Va base a sup } \\
\text { Vb }\end{array}$ & $-75-93$ & E. chinchilloides & & & 1 & 1 & 3 & 1 \\
\hline $\mathrm{Vb}$ & $-100-108$ & C. magellanicus & & 3 & 1 & 2 & 16 & 10 \\
\hline $\mathrm{Vb}$ & $-100-108$ & E. chinchilloides & & & & [1] & 11 & $16[1]$ \\
\hline $\mathrm{Vb}$ & $-100-108$ & O. cuniculus & & & [3] & [3] & [2] & {$[1]$} \\
\hline $\mathrm{Vb}$ & $-100-108$ & R. auritus & & & $3[1]$ & [2] & & [2] \\
\hline $\mathrm{Vb}$ & $-135-145$ & C. magellanicus & & & & & & 1 \\
\hline $\mathrm{Vb}$ & $-135-145$ & E. chinchilloides & & & 2 & & 1 & 1 \\
\hline $\mathrm{Vb}-\mathrm{VI}$ & & C. magellanicus & & & & & & {$[1]$} \\
\hline $\mathrm{Vb}-\mathrm{VI}$ & & E. chinchilloides & & & & [1] & & \\
\hline $\mathrm{Vb}-\mathrm{VI}$ & & R. auritus & & & & {$[2]$} & & [1] \\
\hline VI & $-170-175$ & C. magellanicus & & & & & 1 & \\
\hline VI & $-170-175$ & Eligmodontia sp. & & & & & 1 & \\
\hline VI & $-170-175$ & E. chinchilloides & & & 5 & 4 & 2 & 3 \\
\hline VI & -185 a roca base & C. magellanicus & & & & & & \\
\hline VI & -185 a roca base & Eligmodontia sp. & & & & 1 & & 1 \\
\hline VI & -185 a roca base & E. chinchilloides & & & 3 & 1 & 5 & 3 \\
\hline
\end{tabular}

